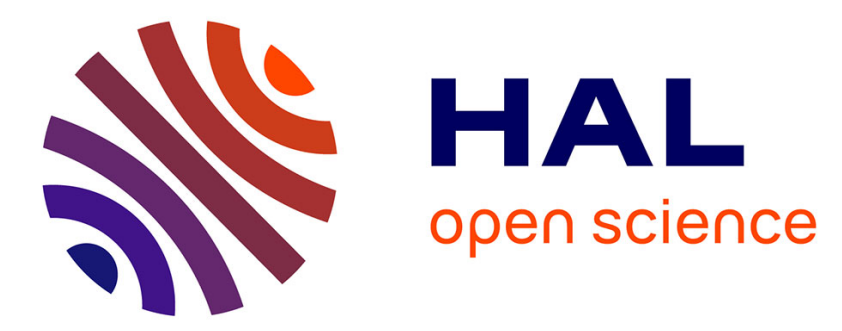

\title{
Numerical homogenisation based on asymptotic theory and model reduction for coupled elastic-viscoplastic damage
}

\author{
Mainak Bhattacharyya, David Dureisseix, Beatrice Faverjon
}

\section{To cite this version:}

Mainak Bhattacharyya, David Dureisseix, Beatrice Faverjon. Numerical homogenisation based on asymptotic theory and model reduction for coupled elastic-viscoplastic damage. International Journal of Damage Mechanics, 2020, 29 (9), pp.1416-1444. 10.1177/1056789520930785 . hal-02866579v2

\section{HAL Id: hal-02866579 \\ https://hal.science/hal-02866579v2}

Submitted on 14 May 2021

HAL is a multi-disciplinary open access archive for the deposit and dissemination of scientific research documents, whether they are published or not. The documents may come from teaching and research institutions in France or abroad, or from public or private research centers.
L'archive ouverte pluridisciplinaire HAL, est destinée au dépôt et à la diffusion de documents scientifiques de niveau recherche, publiés ou non, émanant des établissements d'enseignement et de recherche français ou étrangers, des laboratoires publics ou privés.

\section{(ㅇ)(1) $\$$}

Distributed under a Creative Commons Attribution - NonCommercial - NoDerivatives| 4.0 


\title{
Numerical homogenisation based on asymptotic theory and model reduction for coupled elastic-viscoplastic-damage
}

\author{
Mainak Bhattacharyya $^{1}$, David Dureisseix ${ }^{2}$, and Béatrice Faverjon ${ }^{2}$ \\ ${ }^{1}$ LMT, ENS Paris Saclay, CNRS UMR8535, Université Paris Saclay, F-94235 Cachan \\ CEDEX, France \\ ${ }^{2}$ Univ Lyon, INSA-Lyon, CNRS UMR5259, LaMCoS, F-69621 Villeurbanne CEDEX, \\ France
}

\begin{abstract}
This article deals with damage computation of heterogeneous structures containing locally periodic micro-structures. Such heterogeneous structure is extremely expensive to simulate using classical finite element methods, as the level of discretisation required to capture the micro-structural effects is too fine. The simulation time becomes even higher when dealing with highly non-linear material behaviour, e.g. damage, plasticity and such others. Therefore, a multi-scale strategy is proposed here that facilitates the simulation of non-linear heterogeneous material behaviour in a manner that is computationally feasible. Based on the asymptotic homogenisation theory, this multi-scale technique explores the micro-macro behaviour for elasto-(visco)plasticity coupled with damage. The theory inherently segregates the heterogeneous continua into a macroscopic homogeneous structure, and an underlying heterogeneous microscopic periodic unit cell. Several heterogeneous structures have been simulated using the multi-scale method along with a one-dimensional verification with respect to a reference solution. Additionally, a reduced order modelling is used to prevent large memory requirement for storing micro-structural quantities of interest.

This is a preprint of the article published as: Mainak Bhattacharyya, David Dureisseix and Beatrice Faverjon, Numerical homogenisation based on asymptotic theory and model reduction for coupled elastic-viscoplastic damage, International Journal of Damage Mechanics, Copyright 2020 SAGE Publishing. DOI: 10.1177/1056789520930785.
\end{abstract}

Keywords: asymptotic homogenisation, viscoplasticity, continuum damage, micro-structure, multiscale, reduced order model

\section{Introduction}

Heterogeneous structures like composites, alloys, porous media and such others are common in the engineering world and are used in many applications. This kind of structures generally consists of more than one phase, and the heterogeneity is exhibited at a microscopic scale. Obviously the overall physical behaviour depends on the size, shape, material properties and distribution of the microscopic constituents. A classical finite element simulation for such cases is extremely expensive as the level of discretisation needed to substantiate the underlying micro-heterogeneities will be too fine for real engineering structures. To circumvent this difficulty, multi-scale methods are being developed over the years.

One of the classical multi-scale methods is the self-consistent scheme first proposed in [8] which has later been developed further in [24, 28, 5] and many more like [22]. The main philosophy is the analytical approximation of material properties for the case of a spherical or an ellipsoidal inclusion consisting of one material inside an infinite matrix made of another material. This type of concept has been later used effectively to develop the two-scale damage model, e.g. in [32, 33, 3] for high cycle fatigue simulation.

Another method which has been popular in recent years is the multi-scale computational homogenisation approach or the global-local analysis stemming from the works like [44, 27, 26, 45, 36]. The main philosophy is based on the assumption that the heterogeneous media can be separated into a homogeneous continua and at each material point of it, there exist an underlying heterogeneous microstructure. Constitutive behaviour at the macro-scale is not needed and the macroscopic 
state variables at each integration point can be calculated through homogenisation of the underlying micro-structure. The calculation of the micro-structure can be performed through classical FE approach leading to the well-known $\mathrm{FE}^{2}$ approach, for instance in [9, 10, 37], or using Voronoi cell methods as done in $[20,21]$. Some of the recent developments in the field of $\mathrm{FE}^{2}$ methods can be found in the works like $[17,34,18]$.

There exist another type of multi-scale method, which is based on the asymptotic expansion of the state variables with respect to a scaling factor, see for instance [2, 42]. This type of expansion can be utilised in the governing equations which can then be separated into microscopic and macroscopic problems. The asymptotic homogenisation can provide effective overall structural response including their local microscopic counterparts. The main assumption of asymptotic homogenisation is the quasi-periodicity of the lower length scale. The overall structure is assumed to be an agglomeration of heterogeneous "unit cells", constituting of two or more different materials. A particular unit cell is assumed to be surrounded by identical cells as far as geometric and material properties are concerned. Similar to the $\mathrm{FE}^{2}$ method, the asymptotic homogenisation also sunders the heterogeneous problem into a macroscopic and a microscopic problem, however unlike $\mathrm{FE}^{2}$, behavioural laws exist on both the scales.

Initially this type of methods wa used to solve elastic problems especially for composites in $[12,6]$, for Cosserat media in [15]. A generalisation of the mathematical homogenisation based on eigenstrains for heterogeneous structures was done in [11] for elasto-plasticity, in [14] for non-local brittle damage which was later on extended to include reduced order approximation in [39]. Some other recent application was to find the effect of damage amplification due to micro-crack interaction, see [35], to simulate thermoelectric composites, see [46]. It is noteworthy to mention that most of the analyses are based on $\mathcal{O}(1)$ expansion approaches which results in inaccuracy at regions of high gradients and for low scale separation, higher order homogenisation has been developed for transient dynamics, see [25], elastic composites see [1], and for elasto-plastic heterogeneity see [13].

In a way global-local methods in a philosophical interpretation can be very similar to classical asymptotic homogenisation, i.e. existence of micro-structural periodic unit cells at each material point of the structure. However the non-existence of macroscopic constitutive behaviour for global-local analysis in contrast to separated macro-micro constitutive relations for asymptotic homogenisation can be seen as the main difference. A detailed discussion regarding different numerical homogenisation approaches can be found in [19].

The purpose of this paper is to extend the classical asymptotic developments presented in [6] for typical elasto-(visco)plastic materials coupled with isotropic damage. Usage of damage as a thermodynamically consistent internal variable has been extensive over the years to quantify the loss of load carrying capacity of a structure, the details of which can be found in [31, 29, 30]. Although, the usage of damage in asymptotic homogenisation is not unprecedented, the intent herein is to provide a numerical framework that takes into account Lemaitre-type ductile damage models, i.e. classical elasto-(visco)plasticity coupled with mixed hardening and isotropic damage.

The article begins with the description of the reference problem including the admissibility conditions and constitutive relations. The scale separation procedure that leads into separated micro and macro problems is presented thereafter, followed by the complete numerical strategy with detailed algorithms for solving the separate problems, along with a reduced order strategy. Finally, to exemplify the methodology, a verification study is presented for a one-dimension problem, along with illustrative three-dimensional examples.

\section{Reference problem}

A heterogeneous structure in the domain $\Omega$ as shown in fig. 1 can be considered as a representative media. The boundary $\partial \Omega$ of the domain is divided into $\partial \Omega_{1}$ where a prescribed displacement $U_{d}$ is specified, and the complementary boundary $\partial \Omega_{2}$ where a surface traction $\underline{F}_{d}$ is applied. The heterogeneous media is composed of microscopic locally periodic representative volume elements (RVEs) consisting at least of two different materials. The equilibrium of the structure is then given by

$$
\nabla \cdot \sigma=0 \quad \text { in } \Omega
$$

with the boundary conditions,

$$
\begin{array}{rr}
\underline{F}_{d}=\sigma \cdot \underline{n} & \text { on } \partial \Omega_{2}, \\
\underline{u}=\underline{U}_{d} & \text { on } \partial \Omega_{1} .
\end{array}
$$

The quantity $\sigma$ is the Cauchy stress tensor, $\underline{n}$ being the unit normal at each point on the surface, and $\underline{u}$ is the displacement field. The strain displacement relationship for infinitesimal strain tensor $\varepsilon$ can be written as

$$
\varepsilon=\nabla^{s} \underline{\mathbf{u}} \text { in } \Omega,
$$


with $\nabla^{s} \circ=1 / 2\left[\nabla \circ+(\nabla \circ)^{T}\right]$

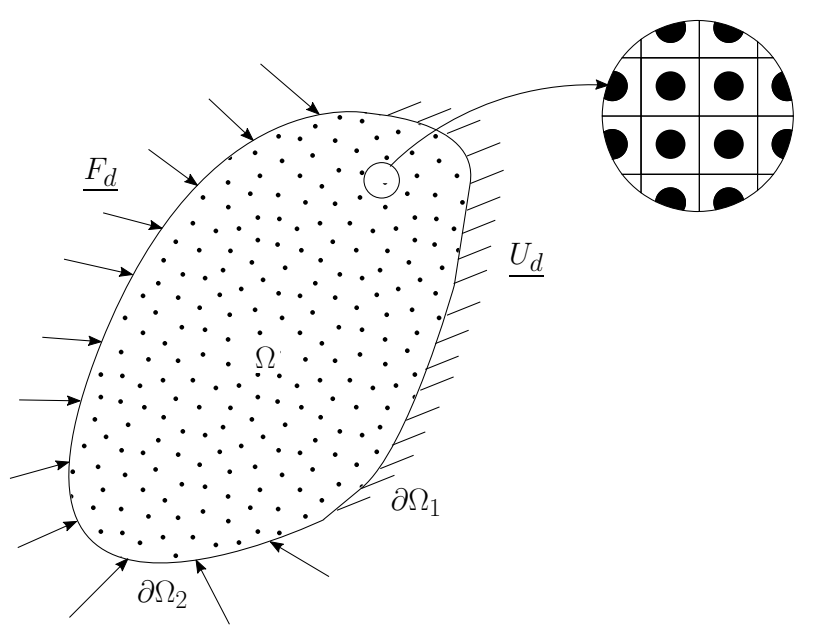

Figure 1: Heterogeneous media in domain $\Omega$ containing locally periodic microstructure.

Apart from such admissibility conditions, the state of the structure at each material point is also described by constitutive equations which are local in space. For the classical viscoplasticity coupled with damage, the extra internal variables involved in the description of the constitutive relations are: $\alpha$ describing the kinematic hardening, $r$ describing the isotropic hardening, and the damage variable $D$. Of course there will exist corresponding dual quantities, namely $\beta, R$, and $Y$. Finally the infinitesimal total strain can be additively decomposed into an elastic part $\varepsilon^{e}$ and an inelastic or plastic part $\varepsilon^{p}$. The equations of state can be written as

$$
\begin{aligned}
\sigma & =\mathbf{C}(1-D) \varepsilon^{e}, \\
\beta & =\mathbf{Q} \alpha, \\
R & =R_{\infty}(1-\exp (-\gamma r)), \\
Y & =\frac{\tilde{\sigma}_{e q}^{2} R_{v}}{2 E} .
\end{aligned}
$$

$\mathbf{C}$ is the Hooke's tensor containing the modulus of elasticity $E$ and Poisson's ratio $\nu$. $\mathbf{Q}$ is a tensor containing material parameter $Q$ that describes kinematic hardening. $R_{\infty}$ and $\gamma$ are material parameters describing isotropic hardening. $R_{v}$ is the triaxiality function which is defined as $R_{v}=(2 / 3)(1+\nu)+3(1-2 \nu)\left(\sigma_{h} / \sigma_{e q}\right)^{2}$, where $\sigma_{h}=(1 / 3) \operatorname{Tr}(\sigma)$ represents the hydrostatic part of the stress tensor, $\sigma_{e q}$ is the von Mises equivalent stress defined as $\sigma_{e q}=\sqrt{(3 / 2) \sigma^{D}: \sigma^{D}}$ with $\sigma^{D}=\sigma-\sigma_{h} \delta$ being the deviatoric stress ( $\delta$ is the unity tensor). The ratio $\sigma_{h} / \sigma_{e q}$ in the definition of $R_{v}$ is called the triaxiality ratio and $\tilde{\sigma}_{e q}$ is the equivalent of the effective stress defined as $\tilde{\sigma}_{e q}=\sqrt{(3 / 2) \sigma^{D} /(1-D): \sigma^{D} /(1-D)}$.

The history dependency of the structure is given by the evolution equations,

$$
\begin{aligned}
\dot{\varepsilon}^{p} & =\frac{\partial f^{p}}{\partial \sigma^{D}} \dot{\lambda} \\
\dot{\alpha} & =-\frac{\partial f^{p}}{\partial \beta} \dot{\lambda} \\
\dot{r} & =-\frac{\partial f^{p}}{\partial R} \dot{\lambda} \\
\dot{D} & =\left(\frac{Y}{S}\right)^{s} \frac{\dot{\lambda}}{1-D} .
\end{aligned}
$$

The viscoplastic multiplier is defined according to Norton's law as,

$$
\dot{\lambda}=k_{v}\left\langle f^{p}\right\rangle^{n_{v}} .
$$

The viscous coefficient $k_{v}$ and viscous exponent $n_{v}$ are material parameters. The von Mises yield function $f^{p}$ delimits the elastic domain and is defined as

$$
f^{p}=\sqrt{\frac{3}{2}\left[\left(\frac{\sigma^{D}}{1-D}-\beta\right):\left(\frac{\sigma^{D}}{1-D}-\beta\right)\right]}-R-\sigma_{y}
$$


with $\sigma_{y}$ being the yield stress.

The numerical solution of such a problem requires extremely fine mesh which will be extremely costly, and for highly non-linear problem, impossible to solve. Therefore a scale separation is necessary to deal with microscopic and macroscopic problem separately.

\section{Scale separation}

Material position vector, at the macroscopic scale $\underline{x}$ (where the structure is considered homogeneous), and at microscopic scale $y$ (describing the heterogeneities), are related to each other by a scaling factor $\xi$ as

$$
\underline{y}=\frac{1}{\xi} \underline{x} .
$$

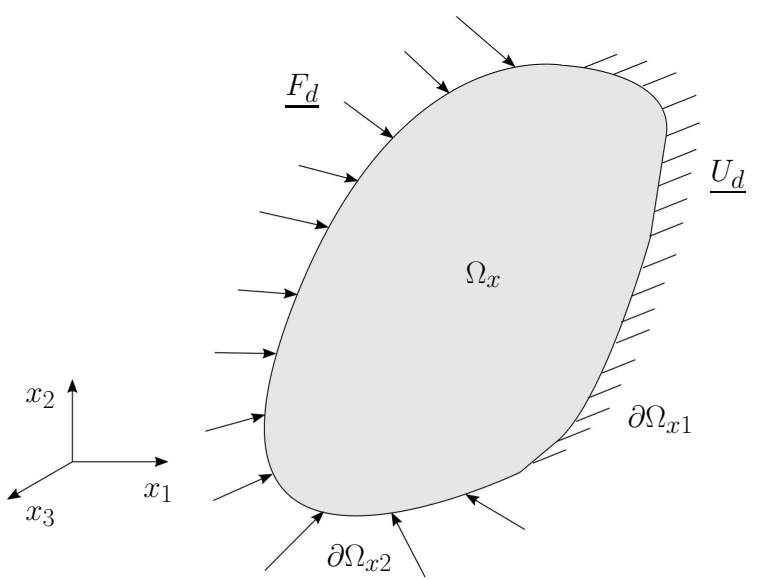

Figure 2: Equivalent homogeneous macroscopic domain.

The parameter $\xi$ has to be as small as possible for better scale separation. Such a separation transforms the macroscopic structural domain into an equivalent homogeneous continua (see fig 2) and defines a heterogeneous RVE (unit cell) over which the averaging is performed (see fig 3 ). It is also assumed that the unit cells are locally periodic or $y$-periodic. This basically means that

$$
\left\langle\nabla_{y} \cdot \chi\right\rangle_{y}=0, \quad \text { and } \quad\left\langle\nabla_{y} \underline{\kappa}\right\rangle_{y}=0
$$

for any tensor field $\chi$ and any vector field $\underline{\kappa}$. The averaging operator $\langle 0\rangle_{y}$ is defined as

$$
\langle\circ\rangle_{y}=\frac{1}{V_{y}} \int_{\Omega_{y}} \mathrm{od} V
$$

where $V_{y}$ is the volume of the unit cell.

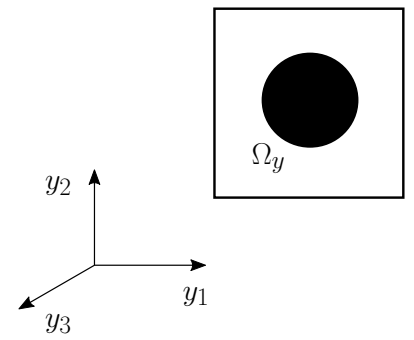

Figure 3: Unit cell.

Using eq. 15 , eqs. 1 and 4 can be rewritten as

$$
\begin{array}{r}
\nabla_{x} \cdot \sigma+\frac{1}{\xi} \nabla_{y} \cdot \sigma=0, \\
\varepsilon=\nabla_{x}^{s} \underline{u}+\frac{1}{\xi} \nabla_{y}^{s} \underline{u} .
\end{array}
$$


with the subscripts $x$ and $y$ represent derivatives with respect to macro and micro scales, respectively. Now the displacement field $\underline{u}$ is expanded with respect $\xi$ as

$$
\underline{u}=\underline{u}_{0}+\xi \underline{u}_{1}+\xi^{2} \underline{u}_{2}+\cdots \text {. }
$$

Using eq. 20 , eq. 19 can be rewritten as

$$
\begin{aligned}
\varepsilon & =\frac{1}{\xi} \nabla_{y}^{s} \underline{u}_{0}+\left(\nabla_{y}^{s} \underline{u}_{1}+\nabla_{x}^{s} \underline{u}_{0}\right)+\xi\left(\nabla_{y}^{s} \underline{u}_{2}+\nabla_{x}^{s} \underline{u}_{1}\right)+\cdots \\
& =\frac{1}{\xi} \varepsilon_{-1}+\varepsilon_{0}+\xi \varepsilon_{1}+\cdots
\end{aligned}
$$

which basically means that the strain tensor also follows an asymptotic series. Of course it has to be noted that the terms in these asymptotic series are $y$ - periodic. Eqs. 20 and 21 are valid for any generic case for infinitesimal strain theory, however the complete resolution of a given mechanical problem rests on the constitutive behaviour governing the state variables. In the following sections this resolution strategy is detailed first for linear cases and then for non-linear cases.

\subsection{An elastic case}

For a linear elastic problem the complete constitutive relation is described by

$$
\sigma=\mathbf{C} \varepsilon
$$

which can be rewritten using eq. 21 as

$$
\begin{aligned}
\sigma & =\frac{1}{\xi} \mathbf{C} \varepsilon_{-1}+\mathbf{C} \varepsilon_{0}+\xi \mathbf{C} \varepsilon_{1}+\cdots \\
& =\frac{1}{\xi} \sigma_{-1}+\sigma_{0}+\xi \sigma_{1}+\cdots
\end{aligned}
$$

This expansion of the stress can be used to rewrite eq. 18 as

$$
\frac{1}{\xi^{2}} \nabla_{y} \cdot \sigma_{-1}+\frac{1}{\xi}\left(\nabla_{x} \cdot \sigma_{-1}+\nabla_{y} \cdot \sigma_{0}\right)+\left(\nabla_{x} \cdot \sigma_{0}+\nabla_{y} \cdot \sigma_{1}\right)+\cdots=0
$$

The idea hereafter is to equate terms with equal powers of $\xi$.

$(-2)$ order problem: equating terms containing $\xi^{-2}$

$$
\nabla_{y} \cdot \sigma_{-1}=0 \quad \text { with } \quad \sigma_{-1}=\mathbf{C} \varepsilon_{-1} \quad \text { and } \quad \varepsilon_{-1}=\nabla_{y}^{s} \underline{u}_{0} .
$$

This immediately provides $\underline{u}_{0}(\underline{x})$ which can be seen as the macroscopic displacement $\underline{u}_{M}$ and $\varepsilon_{-1}=\sigma_{-1}=0$.

$(-1)$ order problem: equating terms containing $\xi^{-1}$

$$
\nabla_{y} \cdot \sigma_{0}=0 \quad \text { with } \quad \sigma_{0}=\mathbf{C} \varepsilon_{0} \quad \text { and } \quad \varepsilon_{0}=\nabla_{y}^{s} \underline{u}_{1}+\nabla_{x}^{s} \underline{u}_{0} .
$$

The term $\nabla_{x}^{s} u_{0}$ is basically the macroscopic strain $\varepsilon_{M}$ and eq. 26 can be seen as the microscopic problem, with the macroscopic strain as a parameter.

0 order problem: equating terms containing $\xi^{0}$

$$
\nabla_{x} \cdot \sigma_{0}+\nabla_{y} \cdot \sigma_{1}=0
$$

Now applying the homogenisation operator, eq. 27 transforms into

$$
\nabla_{x} \cdot\left\langle\sigma_{0}\right\rangle_{y}=0
$$

where $\left\langle\sigma_{0}\right\rangle_{y}$ can be considered as the macroscopic stress $\sigma_{M}$. The boundary conditions can then be written as

$$
\begin{aligned}
& \underline{F}_{d}=\sigma_{M} \cdot \underline{n} \quad \text { on } \partial \Omega_{x 2} \text {, } \\
& \underline{u}_{M}=\underline{U}_{d} \quad \text { on } \partial \Omega_{x 1} .
\end{aligned}
$$

Therefore it is clear that the resolution of eqs. 28, 29, 30, along with the macroscopic constitutive behaviour

$$
\left\langle\sigma_{0}\right\rangle_{y}=\left\langle\mathbf{C} \varepsilon_{0}\right\rangle_{y},
$$

will provide the macroscopic quantities of interest.

In the preceding developments, approximations till the zero order is considered, and it is sufficient as both the microscopic and macroscopic behaviours are captured. This linear case can be solved by calculating unit-cell in an off-line phase and in the on-line phase only the macro problem has to be solved by classical FE, which can then be used to obtain micro-structural variables through post-processing. However, for non-linear case, micro-structures cannot be solved easily in an off-line phase, hence iterative procedures (iterations between macro-micro boundary value problems) are needed as elucidated in the next section. 


\subsection{Extension for elastic-viscoplastic-damage}

To start off, it is assumed first of all that the displacement field is approximated as

$$
\underline{u} \approx \underline{u}_{0}+\xi \underline{u}_{1}+\mathcal{O}\left(\xi^{2}\right) .
$$

For all the other state variables (e.g. stress, strain, etc.) the nullity of the -1 terms are considered to be still valid and it is also considered that the zero order approximation is enough (the subscript 0 will be henceforth omitted for simplicity). For a given set of state variables $\varpi=\left\{\sigma, \varepsilon, \varepsilon^{e}, \varepsilon^{p}, \beta, \alpha, R, r, Y, D\right\}, \varpi$ can be written as

$$
\varpi=\varpi_{M}+\varpi^{*},
$$

where $\varpi_{M}=\langle\varpi\rangle_{y}$ is the macroscopic quantity and $\varpi^{*}=\varpi-\varpi_{M}$ represents its residual. Comparing eq. 21 with eq. 33 , it is clear that

$$
\begin{aligned}
& \varepsilon_{M}=\nabla_{x}^{s} \underline{u}_{0} \\
& \varepsilon^{*}=\nabla_{y}^{s} \underline{u}_{1} .
\end{aligned}
$$

Eqs. 5, 6, 7, 8 can now be written as

$$
\begin{array}{r}
\sigma_{M}+\sigma^{*}=\mathbf{C}\left(1-D_{M}-D^{*}\right)\left(\varepsilon_{M}^{e}+\varepsilon^{e *}\right), \\
\beta_{M}+\beta^{*}=\mathbf{Q}\left(\alpha_{M}+\alpha^{*}\right), \\
R_{M}+R^{*}=R_{\infty}\left(1-\exp \left(-\gamma\left(r_{M}+r^{*}\right)\right)\right), \\
Y_{M}+Y^{*}=\mathcal{G}\left(\sigma_{M}+\sigma^{*}, D_{M}+D^{*}\right) .
\end{array}
$$

with the function $\mathcal{G}$ defined by eq. 8 .

Eqs. 9, 10, 11, 12 can similarly be written as

$$
\begin{array}{r}
\dot{\varepsilon}_{M}^{p}+\dot{\varepsilon}^{p *}=\mathcal{H}\left(\sigma_{M}+\sigma^{*}, \beta_{M}+\beta^{*}, R_{M}+R^{*}, D_{M}+D^{*}\right), \\
\dot{\alpha}_{M}+\dot{\alpha}^{*}=\mathcal{I}\left(\sigma_{M}+\sigma^{*}, \beta_{M}+\beta^{*}, R_{M}+R^{*}, D_{M}+D^{*}\right), \\
\dot{r}_{M}+\dot{r}^{*}=\mathcal{J}\left(\sigma_{M}+\sigma^{*}, \beta_{M}+\beta^{*}, R_{M}+R^{*}, D_{M}+D^{*}\right), \\
\dot{D}_{M}+\dot{D}^{*}=\mathcal{K}\left(\sigma_{M}+\sigma^{*}, \beta_{M}+\beta^{*}, R_{M}+R^{*}, D_{M}+D^{*}, Y_{M}+Y^{*}\right) .
\end{array}
$$

with the functions $\mathcal{H}, \mathcal{I}, \mathcal{J}, \mathcal{K}$ being defined in accordance with eqs. 9, 10, 11, 12 . Now based on the decomposition of the state variables into a homogenised (macroscopic) part and a residual (microscopic) part (eq. 33), the boundary value problem is also decomposed into a homogenised part and a residual part.

Homogenised problem. Following the developments of the elastic problem, the admissibility conditions for the homogenised problem can be written as

$$
\begin{array}{r}
\nabla_{x} \cdot \sigma_{M}=0 \quad \text { in } \Omega_{x}, \\
\underline{F}_{d}=\sigma_{M} \cdot \underline{n} \quad \text { on } \partial \Omega_{x 2}, \\
\underline{u}_{0}=\underline{U}_{d} \quad \text { on } \partial \Omega_{x 1}, \\
\varepsilon_{M}=\nabla_{x}^{s} \underline{\mathbf{u}}_{0} \quad \text { in } \Omega_{x} .
\end{array}
$$

Using the averaging operator, the constitutive relation can be written as,

$$
\begin{array}{r}
\sigma_{M}=\left\langle\mathbf{C}\left(1-D_{M}-D^{*}\right)\left(\varepsilon_{M}^{e}+\varepsilon^{e *}\right)\right\rangle_{y}, \\
\beta_{M}=\left\langle\mathbf{Q}\left(\alpha_{M}+\alpha^{*}\right)\right\rangle_{y}, \\
R_{M}=\left\langle R_{\infty}\left(1-\exp \left(-\gamma\left(r_{M}+r^{*}\right)\right)\right)\right\rangle_{y}, \\
Y_{M}=\left\langle\mathcal{G}\left(\sigma_{M}+\sigma^{*}, D_{M}+D^{*}\right)\right\rangle_{y}, \\
\dot{\varepsilon}_{M}^{p}=\left\langle\mathcal{H}\left(\sigma_{M}+\sigma^{*}, \beta_{M}+\beta^{*}, R_{M}+R^{*}, D_{M}+D^{*}\right)\right\rangle_{y}, \\
\dot{\alpha}_{M}=\left\langle\mathcal{I}\left(\sigma_{M}+\sigma^{*}, \beta_{M}+\beta^{*}, R_{M}+R^{*}, D_{M}+D^{*}\right)\right\rangle_{y}, \\
\dot{r}_{M}=\left\langle\mathcal{J}\left(\sigma_{M}+\sigma^{*}, \beta_{M}+\beta^{*}, R_{M}+R^{*}, D_{M}+D^{*}\right)\right\rangle_{y}, \\
\dot{D}_{M}=\left\langle\mathcal{K}\left(\sigma_{M}+\sigma^{*}, \beta_{M}+\beta^{*}, R_{M}+R^{*}, D_{M}+D^{*}, Y_{M}+Y^{*}\right)\right\rangle_{y} .
\end{array}
$$

The resolution of this will provide the homogenised quantities of interest $\varpi_{M}$. 
Residual problem. The residual admissibility conditions can be written as

$$
\begin{array}{cc}
\nabla_{y} \cdot \sigma^{*}=0 & \text { in } \Omega_{y}, \\
\varepsilon^{*}=\nabla_{y}^{s} \underline{u}_{1} & \text { in } \Omega_{y},
\end{array}
$$

with the only constrained is $\underline{u}_{1}$ has to be periodic in the unit cell. The constitutive behaviour is given by

$$
\begin{array}{r}
\sigma^{*}=\mathbf{C}\left(1-D_{M}-D^{*}\right)\left(\varepsilon_{M}^{e}+\varepsilon^{e *}\right)-\sigma_{M}, \\
\beta^{*}=\mathbf{Q}\left(\alpha_{M}+\alpha^{*}\right)-\beta_{M}, \\
R^{*}=R_{\infty}\left(1-\exp \left(-\gamma\left(r_{M}+r^{*}\right)\right)\right)-R_{M}, \\
Y^{*}=\mathcal{G}\left(\sigma_{M}+\sigma^{*}, D_{M}+D^{*}\right)-Y_{M}, \\
\dot{\varepsilon}^{p *}=\mathcal{H}\left(\sigma_{M}+\sigma^{*}, \beta_{M}+\beta^{*}, R_{M}+R^{*}, D_{M}+D^{*}\right)-\dot{\varepsilon}_{M}^{p}, \\
\dot{\alpha}^{*}=\mathcal{I}\left(\sigma_{M}+\sigma^{*}, \beta_{M}+\beta^{*}, R_{M}+R^{*}, D_{M}+D^{*}\right)-\dot{\alpha}_{M}, \\
\dot{r}^{*}=\mathcal{J}\left(\sigma_{M}+\sigma^{*}, \beta_{M}+\beta^{*}, R_{M}+R^{*}, D_{M}+D^{*}\right)-\dot{r}_{M}, \\
\dot{D}^{*}=\mathcal{K}\left(\sigma_{M}+\sigma^{*}, \beta_{M}+\beta^{*}, R_{M}+R^{*}, D_{M}+D^{*}, Y_{M}+Y^{*}\right)-\dot{D}_{M},
\end{array}
$$

the resolution of which will provide the residual quantities $\varpi^{*}$.

It can be noticed that the homogenised and the residual problems are not decoupled from each other. The interdependence of the two problems means that they are solved using fixed point methods. The procedure begins with an assumed solution $\varpi^{*}$, using which the homogenised problem is solved to obtain $\varpi_{M}$ which will then be used to solve the residual problem to obtain the updated $\varpi^{*}$. This procedure continues till a convergence on a relative error is obtained.

It can also be noticed that no direct macroscopic behaviour is used in this approach: the evolutions of the macroscopic internal variables (defined as averages) are derived from their microscopic counterparts in the underlying micro fields beneath each macroscopic material point.

\section{Numerical implementation}

From an implementation point of view, each Gauss point of the macroscopic structure, represents a micro structural unit cell, i.e. the number of residual problems to be solved is directly proportional to the level of discretisation of the macro-structure. For the macroscopic problem, a classical elasticpredictor plastic-corrector type method is applied to solve macroscopic constitutive behaviour in a global Newton type algorithm for the satisfaction of the equilibrium. It has to be noted that the right-hand-sides of the constitutive equations are averaged quantities of the full micro-structure at each macro Gauss point. The constitutive behaviour at each integration point for a given time step is independent of each other, and hence can be parallelised. This idea of parallelism should also be used while solving the residual problem, where each micro-structure is independent from the other (at the micro-level) and hence the residual problems can be solved using parallel cores.

A classical time incremental procedure is used to calculate $\varpi_{i}$ at time step $t_{i}$, knowing $\varpi_{i-1}$, i.e. at $t_{i-1}$. The initial values at $t_{0}$ is considered to be zero, and thereafter at each subsequent time step $t_{i}$, the algorithm starts with initial guesses of $\varpi_{i}^{*}=\varpi_{i-1}^{*}$ (see algorithm 3).

\subsection{Solution of the homogenised problem at $t_{i}$}

The basic methodology is based on classical Newton type framework that ensures satisfaction of the macroscopic equilibrium. For the non-linear material behaviour, the classical elastic predictorviscoplastic corrector scheme is used.

All the quantities, i.e. $\varpi_{M i}$ and $u_{M i}$ are initialised from the previous time step $t_{i-1}$, after that a global Newton algorithm is employed as shown in algorithm 1. It is evident that this type of framework is quite standard, however what is interesting is that the trial yield function is calculated at every microscopic integration point for a given macroscopic Gauss point. The corrector step for a particular macroscopic Gauss point, is employed if any of the underlying microscopic Gauss point is inelastic. The solution of the set of non-linear equations can be achieved by using any standard non-linear solvers like Newton-Raphson or trust-region algorithms. The numerical tangent $\mathbf{C}_{T}$ can be calculated from the Jacobian of their corresponding linearised form. The for loop used for every macroscopic integration point has to be parallelised as they are independent of each other, for the sake of computational time gain.

Once $\varpi_{M i}$ is obtained the next step is to solve the residual problem. 


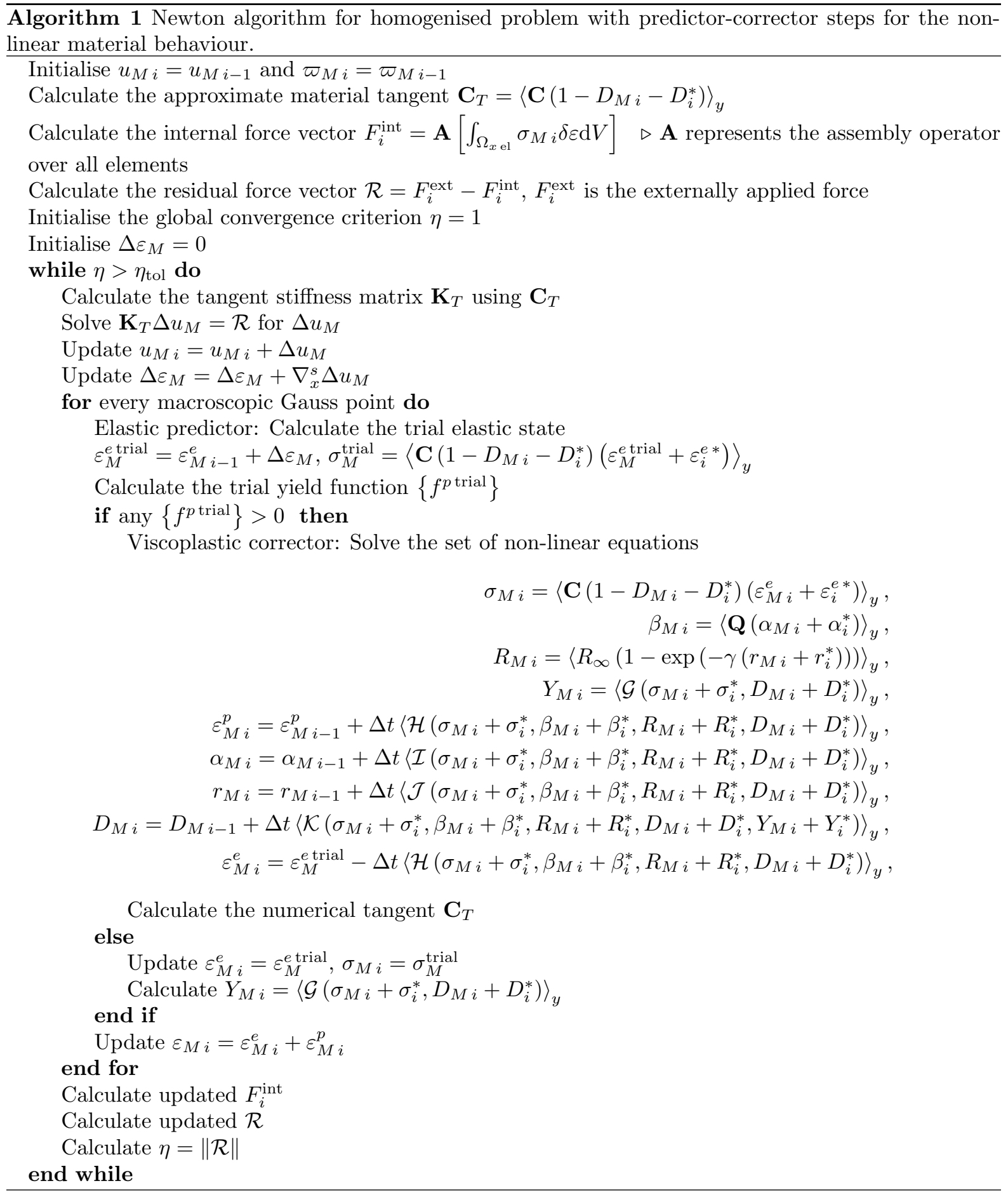




\subsection{Solution of the residual problem at $t_{i}$}

This will be the most time consuming part of the whole simulation as a large number of microstructural problems need to be calculated (same as the number of macro Gauss points). At this particular level, the micro-problems are inherently decoupled hence they can be parallelised.

For a given micro-structural problem, one important aspect is to maintain periodicity of the displacements, strains, stresses and other state variables, see [43], also the anti-periodicity of the traction should be maintained. Without going into details it is safe to say that if a periodicity on displacement is maintained, all the other cases will be satisfied. For a one dimensional microstructure it is easily achieved by clamping the two extremities. For a two dimensional case, the opposite edges need to be periodic, i.e. the nodal degrees of freedom (DOFs) should be same on the periodic edges. Any two adjacent edges can be chosen as master edges and their corresponding opposite edges become the slave edges. For a three dimensional unit cell there will be three master surfaces corresponding to three opposite slave surfaces. There will also be three master edges, each of which will have three slave edges. Of course, periodic boundary conditions do not restrict rigid body motions hence the corners should be clamped to avoid them.

The idea thereby is while solving the $\mathrm{FE}$ equation i.e. $\mathbf{K}_{T} \Delta u=\mathcal{R}$, the corresponding masterslave pair should have the same value. This can be achieved by reducing the linear system by excluding the slave DOFs. A substitution matrix $\mathbf{G}$ can be constructed that can perform this operation, see [16]. This matrix can be used to reduce the linear system as $\mathbf{K}_{T \text { red }} \Delta u_{\text {red }}=\mathcal{R}_{\text {red }}$, where $\mathbf{K}_{T \text { red }}=\mathbf{G}^{T} \mathbf{K}_{T} \mathbf{G}, \mathcal{R}_{\text {red }}=\mathbf{G}^{T} \mathcal{R}$, and $\Delta u=\mathbf{G} \Delta u_{\text {red }}$.

For a particular micro-structure, the solution is achieved according to algorithm 2. As can be observed it is not a standard one, the predictor step is before the while loop and it avoids the while loop when the macroscopic loading is elastic. Inside the while loop, the non-linear set of equations are always solved, even for a Gauss point which is elastic. The reason behind is the fact that as the macroscopic behaviour at the time step is inelastic, the residual quantities need to be corrected for any elastic micro Gauss point.

Once $\varpi_{i}^{*}$ is calculated the algorithm once again solves for $\varpi_{M i}$ and so on (see algorithm 3 for better elucidation). Such a staggered scheme requires an error indicator which for this case at its $j_{\text {th }}$ iteration is defined in terms of stress as

$$
\iota=\left(\frac{\sum_{\Omega_{y} \times[0, T]}\left(\sigma_{i}^{j}-\sigma_{i}^{j-1}\right):\left(\sigma_{i}^{j}-\sigma_{i}^{j-1}\right) \mathrm{d} V_{y} \mathrm{~d} t}{\sum_{\Omega_{y} \times[0, T]}\left(\sigma_{i}^{j}+\sigma_{i}^{j-1}\right):\left(\sigma_{i}^{j}+\sigma_{i}^{j-1}\right) \mathrm{d} V_{y} \mathrm{~d} t}\right)^{1 / 2} .
$$

The stress used in eq. 65 is the total stress (sum of the homogenised and the residual) and the integrals are summed for all the computed micro-structures.

\subsection{Reduced order modelling for storage compression}

It is quite obvious that for each time step $t_{i}$ all the quantities for all the micro-structures need to be stored to progress to the next time step. The memory consumption for such cases can be huge and a reduced order strategy can be implemented to overcome this difficulty. Indeed, the use of surrogate micro models for non-linear homogenisation is appealing and different approaches can be used depending on the material behaviour model, e.g. in [38, 40, 23]. It has also been used in the context of time homogenization, but without concerning damage and fatigue in [41].

Herein, the basic idea for a particular quantity of interest is to have a single set of basis vectors representing all the micro-structures and their difference in behaviour will be represented by the time function which is a scalar and defined at each macroscopic Gauss point.

At time step $t_{i}$ and under the macro Gauss point number $n$, let $\chi_{i}^{n}$ denote any of the micro state fields in the set $\left\{u_{1}^{n}, \varpi_{i}^{*, n}\right\}$. Let us assume that there are $m$ basis orthogonal vectors needed to describe $\chi_{i-1}^{n}$, i.e.

$$
\chi_{i-1}^{n}=\sum_{k=1}^{m} \mathcal{U}^{k} \mathcal{A}_{i-1}^{k, n},
$$

where $\mathcal{U}^{k}, k=1, \ldots, m$, are the micro spatial basis vectors (independent of $n$ ), and the corresponding amplitudes are given by the time functions $\mathcal{A}^{k, n}\left(t_{i-1}\right)=\mathcal{A}_{i-1}^{k, n}$. Additionally, we consider normalized basis vectors: $\left\|\mathcal{U}^{k}\right\|^{2}=\left\langle\mathcal{U}^{k}, \mathcal{U}^{k}\right\rangle=1$ where $\langle\circ, \circ\rangle$ represents inner product.

There are at least two strategies for updating the basis. If the number of macro Gauss points is not too large, the first approach gathers the micro fields and makes use of a singular value decomposition (SVD):

$$
\chi_{i}=\left[\chi_{i}^{1} \ldots \chi_{i}^{n} \ldots\right]=\mathcal{V B} .
$$




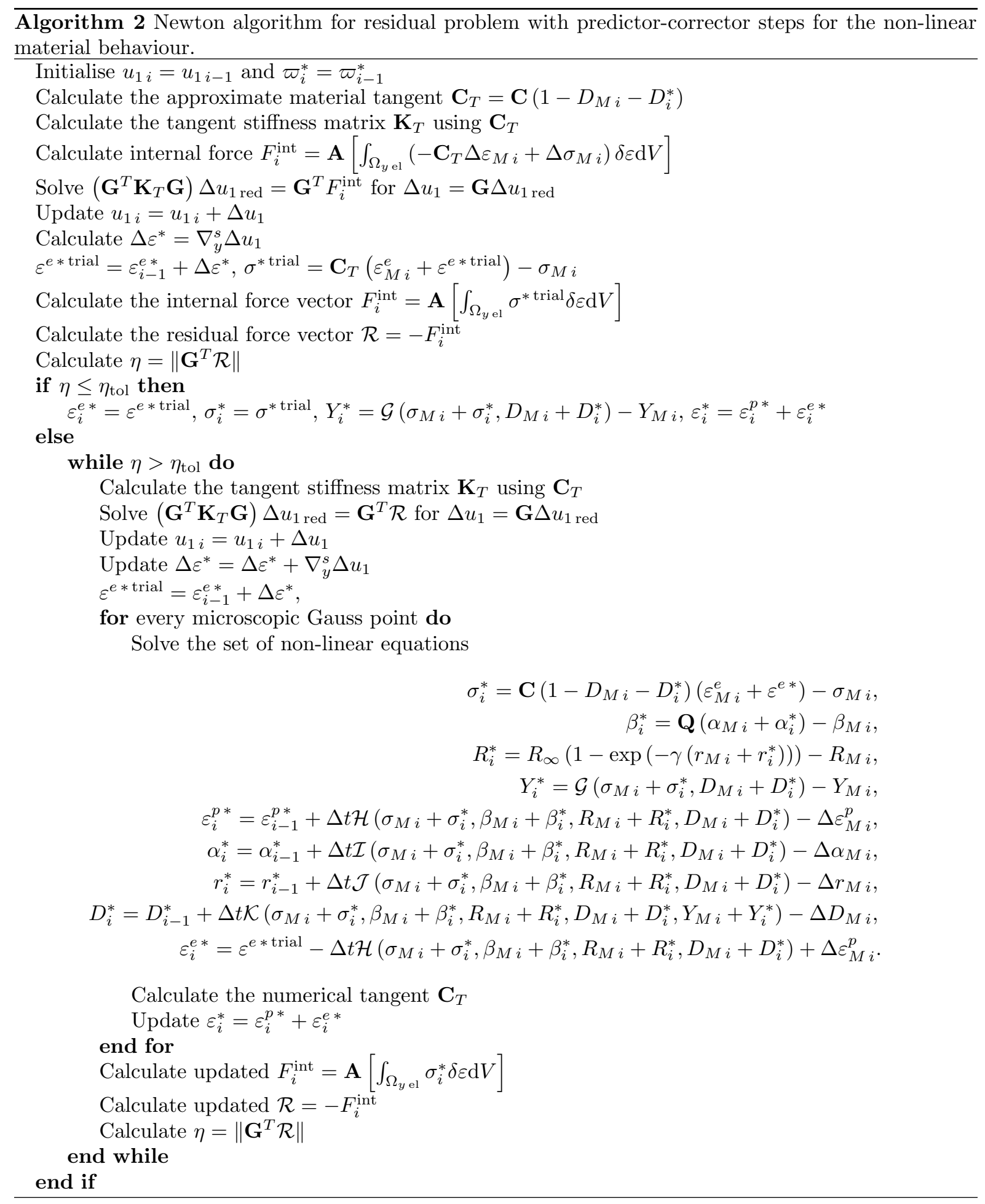




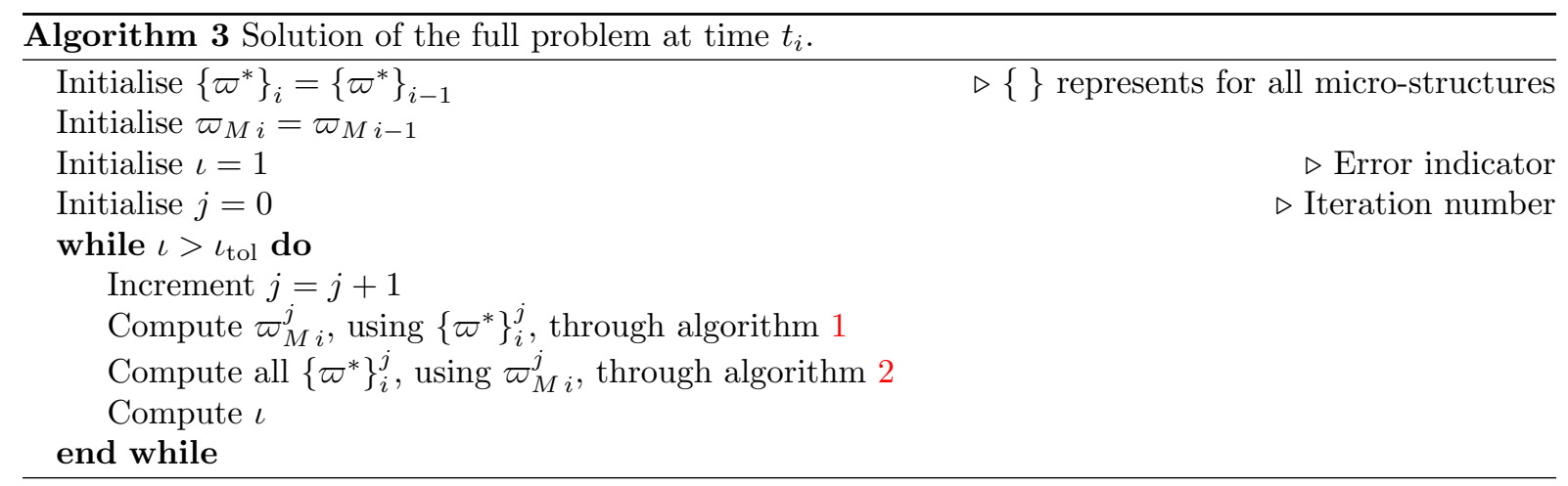

to produce a potential new basis vector $\mathcal{V}$. To simplify, consider the case where only 1 vector is contained in $\mathcal{V}$ (rank-1 decomposition); eq. 67 can then be written as

$$
\begin{aligned}
\chi_{i}^{n} & =\left(\mathcal{V}-\sum_{k=1}^{m}\left\langle\mathcal{U}^{k}, \mathcal{V}\right\rangle \mathcal{U}^{k}\right) \mathcal{B}+\sum_{k=1}^{m}\left\langle\mathcal{U}^{k}, \mathcal{V}\right\rangle \mathcal{U}^{k} \mathcal{B} \\
& =\mathcal{U}^{m+1} \mathcal{A}_{i}^{m+1, n}+\sum_{k=1}^{m} \mathcal{U}^{k} \mathcal{A}_{i}^{k, n}
\end{aligned}
$$

where

$$
\begin{aligned}
& \mathcal{U}^{m+1}=\frac{\mathcal{V}-\sum_{k=1}^{m}\left\langle\mathcal{U}^{k}, \mathcal{V}\right\rangle \mathcal{U}^{k}}{\left\|\mathcal{V}-\sum_{k=1}^{m}\left\langle\mathcal{U}^{k}, \mathcal{V}\right\rangle \mathcal{U}^{k}\right\|}, \\
& \mathcal{A}_{i}^{m+1, n}=\left\|\mathcal{V}-\sum_{k=1}^{m}\left\langle\mathcal{U}^{k}, \mathcal{V}\right\rangle \mathcal{U}^{k}\right\| \mathcal{B}, \\
& \mathcal{A}_{i}^{k, n}=\left\langle\mathcal{U}^{k}, \mathcal{V}\right\rangle \mathcal{B}, \quad k=1, \ldots, m .
\end{aligned}
$$

Now if the magnitude of the $\mathcal{A}_{i}^{m+1, n}$ is sufficiently large then the new pair, whose number is $m+1$, is added to the existing basis, else it is rejected. This procedure is easily generalised for a higher order rank decomposition, when $\mathcal{V}$ is a set of vectors.

Alternatively, a second approach can be used: the micro field $\chi_{i}^{n}$ alone can be used to complete the basis, macro Gauss point after macro Gauss point, by orthogonalisation:

$$
\begin{aligned}
\chi_{i}^{n} & =\left(\chi_{i}^{n}-\sum_{k=1}^{m}\left\langle\mathcal{U}^{k}, \chi_{i}^{n}\right\rangle \mathcal{U}^{k}\right)+\sum_{k=1}^{m}\left\langle\mathcal{U}^{k}, \chi_{i}^{n}\right\rangle \mathcal{U}^{k} \\
& =\mathcal{U}^{m+1} \mathcal{A}_{i}^{m+1, n}+\sum_{k=1}^{m} \mathcal{U}^{k} \mathcal{A}_{i}^{k, n}
\end{aligned}
$$

and we proceed similarly as before.

In the following, the first approach is used for numerical results.

It is quite obvious that the time functions are basically dependent on $n$, some micro-structures may need more basis vectors to represent $\chi_{i}^{n}$. However as a single set of spatial basis vectors are used for all micro-structures, if an extra basis $m+1$ is added at micro-structure number $n$ to a pre-existing set of $m$ bases, the time functions $\left\{\mathcal{A}_{i}^{m+1, q}\right\}_{q \neq n}$ are added as zero. This will basically give the same number of space-time pairs for all micro-structures at a given time step $t_{i}$.

When new basis vectors are added to the $m$ pre-existing basis vectors, to be consistent, the corresponding previous time functions (for $t<t_{i}$ ) are also added as zero.

The algorithm can start with an arbitrary but normal basis vector and the basis can be enriched at each time step. At each time step $t_{i}$ any needed micro quantity $\chi_{i-1}^{n}$ at the previous time step can be locally reconstructed from eq. 66 .

In a more heuristic sense, this reduced order approximation can be interpreted as an augmented model describing the non-linear material state of the micro-structures. Indeed, the time functions $\mathcal{A}$ are defined at each macroscopic Gauss point and at each time step. This reduced order strategy basically provides the macroscopic equivalent of the state variables (observable or internal) of the underlying micro-structure, and their distributions are given by a single set of spatial basis vectors (for each $\chi$ ). Thereby the state of the micro-structures are captured and stored in a highly reduced memory.

Compared to the $\mathrm{FE}^{2}$ approach, the information interchange between the scales is similar, so the proposed approach is similar (though not exactly same) to $\mathrm{FE}^{2}$ concerning the CPU cost (provided 
that convergence is similar). For linear problems e.g. linear elasticity, it is possible to avoid FE computation on all the micro-structures using off-line pre-computations. Only a single representative unit cell simulation would be sufficient, and linear operator relating the micro and macro quantities can be defined, which basically translates the complete problem into a homogenised problem (quantities at unit cell level can be calculated as post processing through the aforementioned linear operators). For non-linear problems (as targeted herein), no such linear operators can be defined. Instead, the selected solution is to design an iterative procedure between the two scales for obtaining the quantities of interest, both the macroscopic scale and all the underlying micro-structure. In this particular case, asymptotic homogenisation provides an alternative methodology to $\mathrm{FE}^{2}$, which is not necessarily more computationally efficient. Nevertheless, using the micro-macro representation and deriving the micro problem as a correction (and not the full solution), the various micro-cell problems may have stronger similarities one to the other, so the use of ROM is effective to improve the storage cost. Embedding the ROM strategy within the classical $\mathrm{FE}^{2}$ approach for comparison purposes, both on $\mathrm{CPU}$ and storage costs is a direct perspective to this article.

If the augmentation is interpreted with macroscopic fields $\mathcal{A}$ as some new macroscopic internal variables, their meaning and number needs clarification. In a general case, and with a continuum model, the strict equivalence of a macroscopic model to the reference fine-scale problem (i.e. the reference solution is the same as the macroscopic one with re-localisation, when no boundary effects occur), leads to an infinite number of macro internal variables, see [43]. A similar situation resides in viscoelasticity models with an heredity function that tracks back in the past: if the function has to be defined on its full range, it contains an infinity of information, see [4]. As an alternative, a finite number of internal variables, obtained through truncated Prony's series, is a good approximation. As the heredity function is vanishing for long past times, it can be truncated as well, with a good approximation. A similar situation may arise for homogenisation of thermal problems, see [7]. For a discretised problem, the strict equivalence is obtained if the number of macroscopic internal variables is the same as the sum of the number of microscopic internal variables of the cell behind a macroscopic integration point that may still be huge. The model in eqs. 47-54 has a large number of internal variables: due to averaging, and presence of residual quantities, all microscopic internal variables are involved, which can be expected to be truncated as well. The ROM is a good solution to perform this in a data-driven adaptive way (this is why the number of additional internal variables may increase during evolution time); indeed, this is depending on the loading trajectory on the cells (for a pure traction case, the mandatory number of internal variables would be smaller).

\section{A one dimensional verification}

A classic one dimensional example as shown in fig. 4 is chosen for verification of the method. The bar is of length $L=1000 \mathrm{~mm}$ and its area of cross section varies according to a cosine curve such that it is maximum $\left(A_{M}=150 \mathrm{~mm}^{2}\right)$ at $x=0$ and $x=L$, and minimum $\left(A_{m}=100 \mathrm{~mm}^{2}\right)$ at $x=L / 2$. A traction force $F_{d}=1500 \sin (2 \pi t / \mathcal{T}) \mathrm{N}$ is applied for one cycle of time period $\mathcal{T}=10 \mathrm{~s}$ at the right extremity of the bar, while the left one is clamped. The bar is essentially composed of periodic unit cells containing two materials (see fig. 4) $\mathrm{Mat}_{1}$ and $\mathrm{Mat}_{2}$, which are considered to be Cr-Mo steels at $20^{\circ} \mathrm{C}$ and $580^{\circ} \mathrm{C}$, respectively. The corresponding material parameters are given in table 1 , and the loading is such that Mat ${ }_{1}$ remains elastic. The length of the unit cell is $l=1 \mathrm{~mm}$, i.e. the scale ratio is $\xi=10^{-3}$, and half of it comprises of Mat 1 , and Mat M $_{2}$ constitutes the other half.

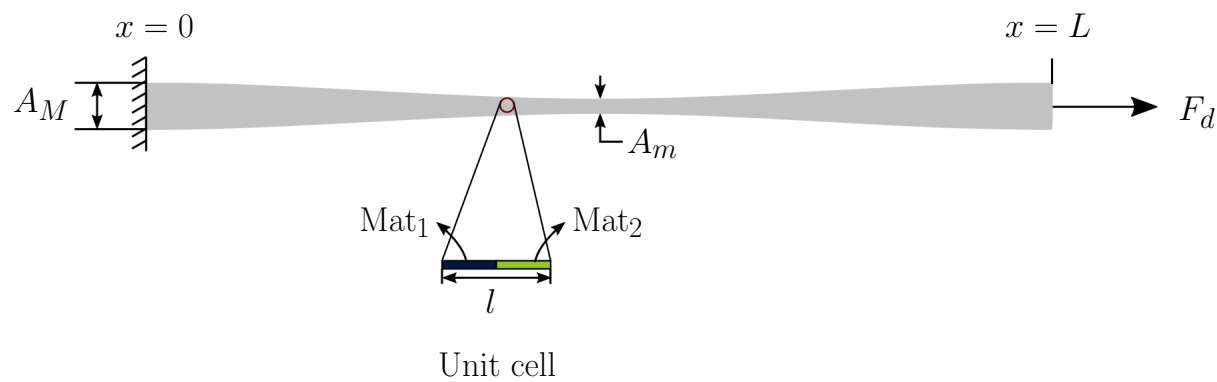

Figure 4: A bar in traction.

To calculate a reference solution, the structure is uniformly discretised using 4000 linear onedimensional isoparametric bar elements having 1 Gauss point per element. The discretisation is such that each RVE is discretised by 4 elements. A classical mono-scale simulation is carried on using Newton type algorithm to ensure global equilibrium and predictor-corrector steps to solve material non-linearities. 
For the multi-scale analysis, the macroscopic structure is discretised using only 50 elements, and the unit cell is discretised using 4 elements. A uniform time step of $0.5 \mathrm{~s}$ is used for both the reference and multi-scale solution. The tolerance that measures the convergence of the Newton algorithm is taken to be $10^{-8}$ for both the macro and the micro problem, and the tolerance of the iterative error criteria $\iota$ is considered to be $10^{-4}$.

Table 1: Material parameters.

\begin{tabular}{lcc}
\hline & Mat $_{1}$ & Mat $_{2}$ \\
\hline$E$ & $200000 \mathrm{MPa}$ & $134000 \mathrm{MPa}$ \\
$\nu$ & 0.3 & 0.3 \\
$\sigma_{y}$ & $180 \mathrm{MPa}$ & $85 \mathrm{MPa}$ \\
$Q$ & $20000 \mathrm{MPa}$ & $6000 \mathrm{MPa}$ \\
$R_{\infty}$ & $6 \mathrm{MPa}$ & $30 \mathrm{MPa}$ \\
$\gamma$ & 2 & 2 \\
$K_{v}$ & $195 \mathrm{MPa} . \mathrm{s}^{1 / n_{v}}$ & $1220 \mathrm{MPa} . \mathrm{s}^{1 / n_{v}}$ \\
$n_{v}$ & 12.5 & 2.5 \\
$S$ & $2.8 \mathrm{MPa}$ & $0.6 \mathrm{MPa}$ \\
$s$ & 2 & 2 \\
\hline
\end{tabular}

An error criteria has been defined as

$$
e r_{\wp}=\left(\frac{\sum_{\Omega_{y} \times[0, T]}\left(\wp_{\mathrm{ref}}-\wp_{\mathrm{ts}}\right) \cdot\left(\wp_{\mathrm{ref}}-\wp_{\mathrm{ts}}\right) \mathrm{d} V \mathrm{~d} t}{\sum_{\Omega_{y} \times[0, T]}\left(\wp_{\mathrm{ref}}+\wp_{\mathrm{ts}}\right) \cdot\left(\wp_{\mathrm{ref}}+\wp_{\mathrm{ts}}\right) \mathrm{d} V \mathrm{~d} t}\right)^{1 / 2},
$$

with $\wp \in\left\{D, \sigma, \varepsilon^{p}\right\}$, and the subscripts "ref" and "ts" correspond to the reference and the two-scale solutions, respectively. The spatial integrations are on every unit cells, and the summation is over all the unit cells. The error on damage is found to be $e r_{D}=0.29 \%$, the error on stress is $e r_{\sigma}=0.0327 \%$, and the error on plastic strain is calculated to be $e r_{\varepsilon} p=0.17 \%$.

The spatial distribution of $\wp$, where the corresponding quantities are maximum for their respective temporal evolutions, is plotted in fig. 5. The maxima are obtained at the centre of the bar, with the minima at the extremities. The curves which have almost zero ordinate for damage and plastic strain represents the undamaged elastic conditions of $\mathrm{Mat}_{1}$ while the non-zero distributions are for $\mathrm{Mat}_{2}$.

For the temporal evolution, the maximum total damaged point (at the micro-scale) is chosen, and errors $\wp$ for this particular point are plotted with respect to time, along with their corresponding reference curves (see fig. 6). It is quite obvious that this point corresponds to $\mathrm{Mat}_{2}$. The contiguity of the multi-scale solution to the reference is quite vivid (as seen in figs. 5, 6).

As far as the CPU time is concerned, the run time of the multi-scale method is $48 \%$ of the run time of the reference problem, i.e. a gain of $52 \%$ is achieved on this unidimensional problem.

For the reduced basis approximation, a total of two basis vectors for each of the quantities of interest are finally obtained to approximate the residual solution. This accounts for a huge reduction in memory consumption for any particular time step. A memory reduction of $85 \%$ per time step is achieved when compared to a multi-scale solution without the reduced order approximation.

\section{A three dimensional simulation for cyclic loading}

An L-shaped structure as shown in fig. 7 is considered for a demonstrative example. The geometry of the structure is quantified by $L_{1}=L_{2}=500 \mathrm{~mm}, W_{1}=W_{2}=100 \mathrm{~mm}$ and $\varrho=100 \mathrm{~mm}$. The structure is clamped at the bottom surface and a surface traction $F_{d}=7.2 \sin (2 \pi t / \mathcal{T}) \mathrm{N} / \mathrm{mm}^{2}$ of time period $\mathcal{T}=10 \mathrm{~s}$ is applied on the right surface for 1 cycle.

The underlying micro-structure shown in fig. 8 consists of a cube of length $l_{1}=l_{2}=l_{3}=0.1 \mathrm{~mm}$. The majority of the cube is made of Mat, while a cylindrical portion of radius $\vartheta=0.025 \mathrm{~mm}$ is composed of $\mathrm{Mat}_{2}$ (see fig. 8).

The macroscopic structure is discretised using 195 linear 8 noded isoparametric hexahedral elements with 8 Gauss points per element. This constitutes of a total of 360 nodes and 1560 Gauss points. For a single micro-structure, 360 elements (of the same type) are used, that results in a total of 534 nodes and 4272 Gauss points. The tolerance measuring the convergence of the Newton algorithm is taken to be $10^{-8}$ for both the macro and the micro problems. The tolerance of the iterative error criteria $\iota$ is considered to be $10^{-4}$. 

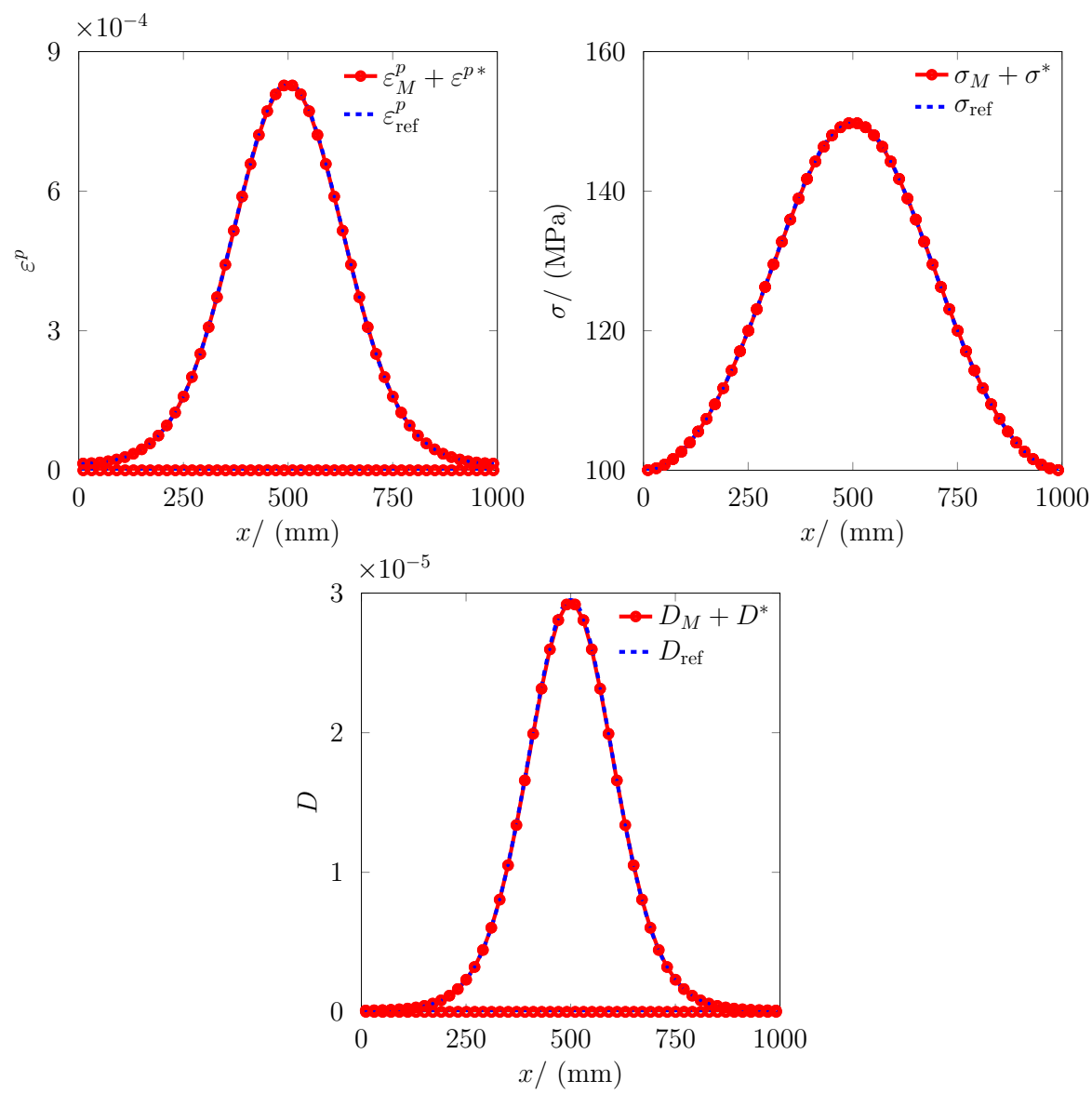

Figure 5: Distribution of damage, stress and plastic strain over the length of the bar. 

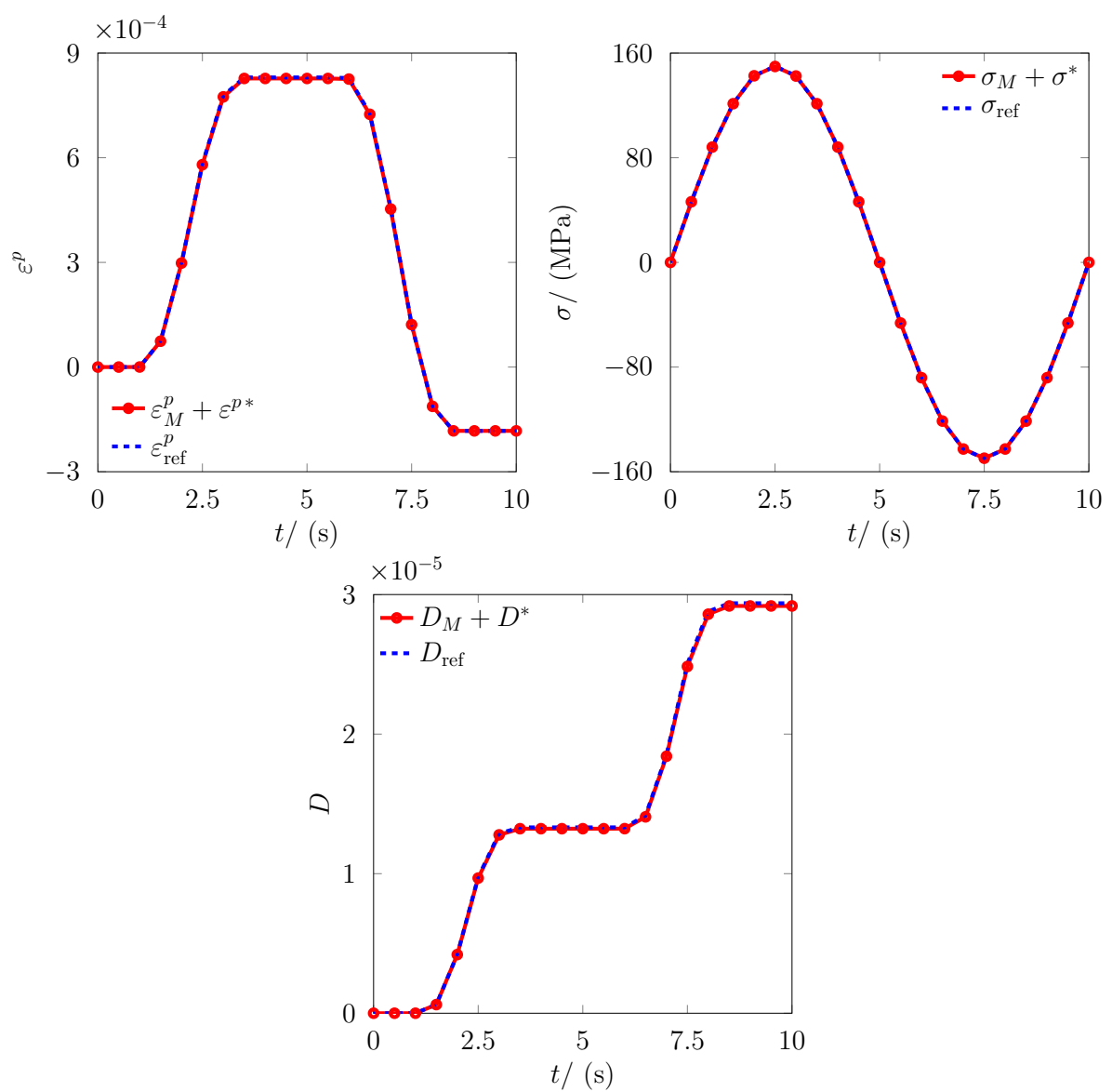

Figure 6: Evolution of damage, stress and plastic strain for Mat ${ }_{2}$ in an RVE (unit cell) located at the centre of the bar.

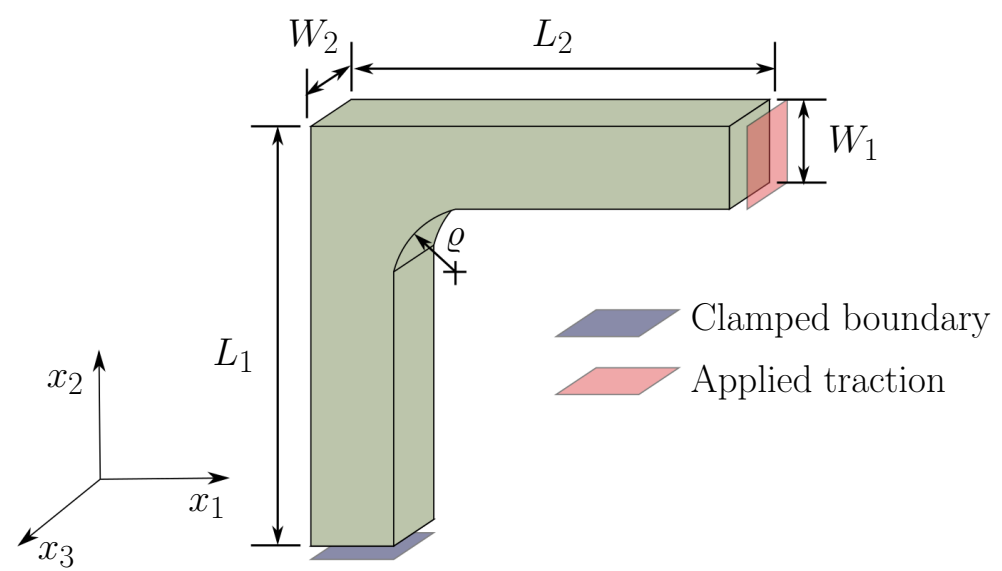

Figure 7: L-shaped structure. 


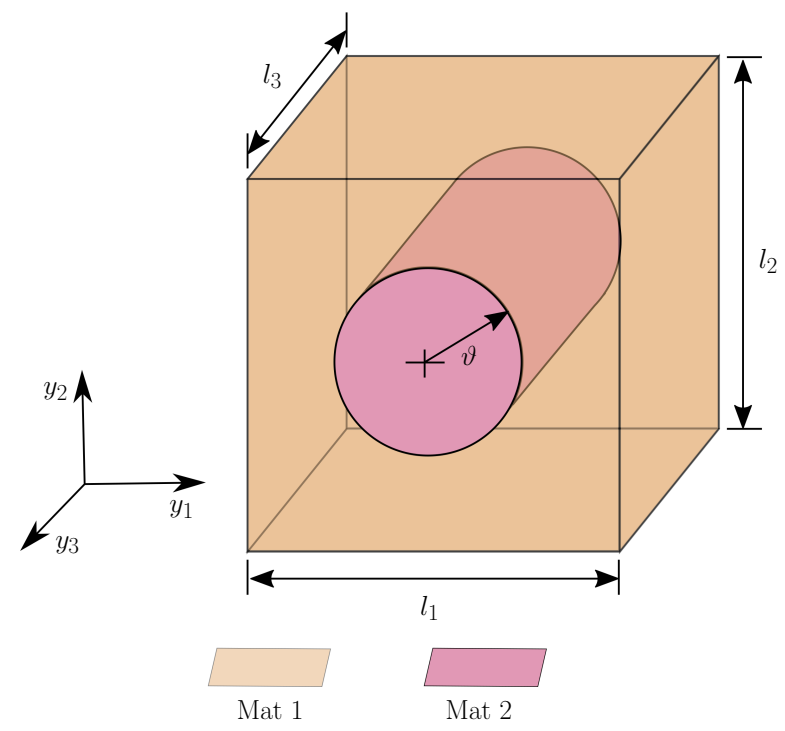

Figure 8: Micro-structural unit cell.
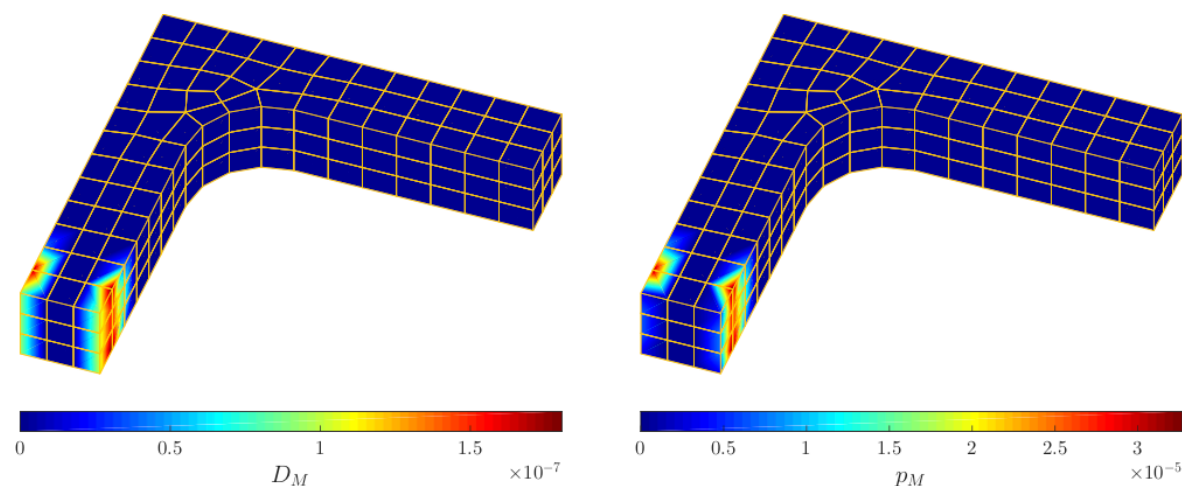

Figure 9: Distribution of macroscopic damage and cumulative plastic strain at the end of loading. 
The distribution of damage and cumulative plastic strain at the end of loading in the macrostructure is shown in fig. 9. The macroscopic cumulative plastic strain is defined here with respect to the $r_{M}$ and $D_{M}$ as

$$
\dot{p}_{M}=\frac{\dot{r}_{M}}{1-D_{M}} .
$$

The corresponding total distribution at the underlying micro-structure of the weakest macroscopic Gauss point is shown in fig. 10, with the residual accumulated plastic strain defined with respect to $r^{*}$ and $D^{*}$ as

$$
\dot{p}^{*}=\frac{\dot{r}^{*}}{1-D^{*}} .
$$

It is assumed herein that as the damage and the isotropic hardening variable follows eq. 33, so does the cumulative plastic strain. It is clear that although the macroscopic quantities indicate damage and plasticity, at the micro-scale only the region of $\mathrm{Mat}_{2}$ shows non-linear behaviour, and the matrix region made of $\mathrm{Mat}_{1}$ is completely elastic and undamaged. This fact is further emphasised in fig. 11, where the homogenised values are much lower than their total counterparts in the fibre region of the unit-cell for a given time. Also the matrix region remains completely undamaged and elastic for the complete loading. As far as the reduced basis approximation is concerned, to approximate $\varepsilon^{p *}$,
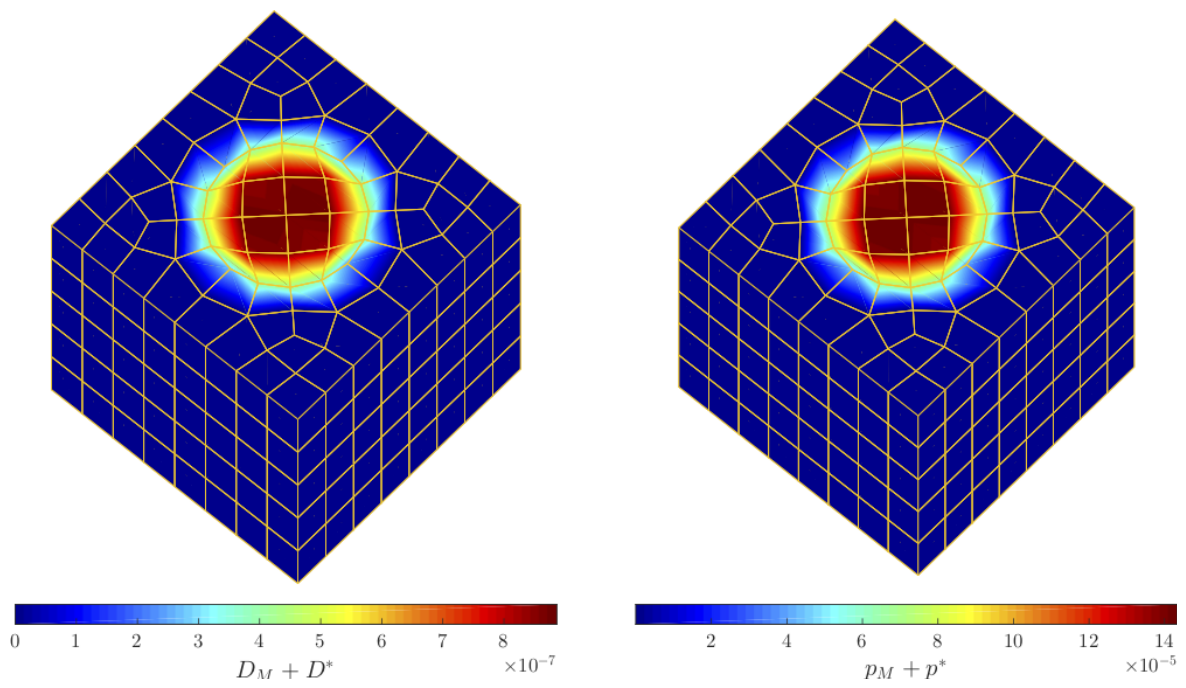

Figure 10: Distribution of total damage and cumulative plastic strain at the end of loading for the weakest micro-structure.

$\alpha^{*}, \beta^{*}, R^{*}, r^{*}, D^{*}$, ten basis vectors for each of them are generated. Seventeen basis vectors each are needed to approximate $\sigma^{*}$ and $Y^{*}$, and seven to approximate $\underline{u}_{1}$.

Although the damage for both the one-dimensional and three-dimensional cases are low, as only one load cycle is considered, these illustrative examples display the effectiveness of the proposed methodology and can be used for fatigue type loading.
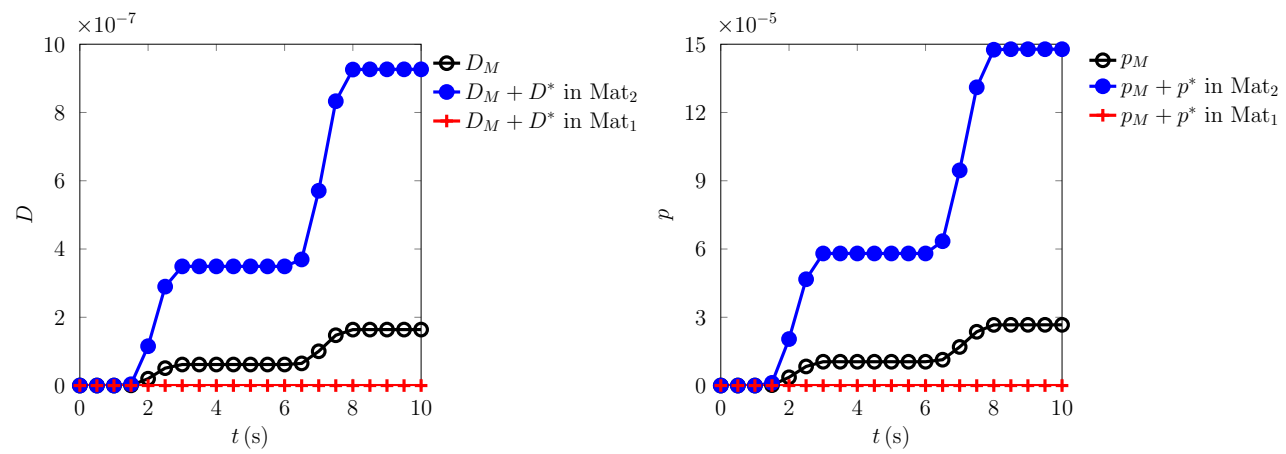

Figure 11: Temporal evolution of damage and cumulative plastic strain. 


\section{$7 \quad$ A three dimensional simulation for monotonic loading}

A square prism is considered with an elliptical through hole as a macroscopic structure as shown in fig. 12. The upper and lower surface of the block is subjected to equal and opposite traction loads. The symmetry of the structure dictates that only one-eighth of the whole block is considered for analysis. The geometry of the symmetric structure is given by $L_{1}=L_{2}=1000 \mathrm{~mm}$, and $L_{3}=200 \mathrm{~mm}$. The geometry of the groove is given by $\varrho_{a}=400 \mathrm{~mm}$ and $\varrho_{b}=200 \mathrm{~mm}$. The surface traction $F_{d}=28.8 \frac{t}{T} \mathrm{~N} / \mathrm{mm}^{2}, \forall t \in[0, T]$, with $T=2 \mathrm{~s}$ is applied on the top surface, and the bottom, left and rear surfaces have symmetric boundary conditions.

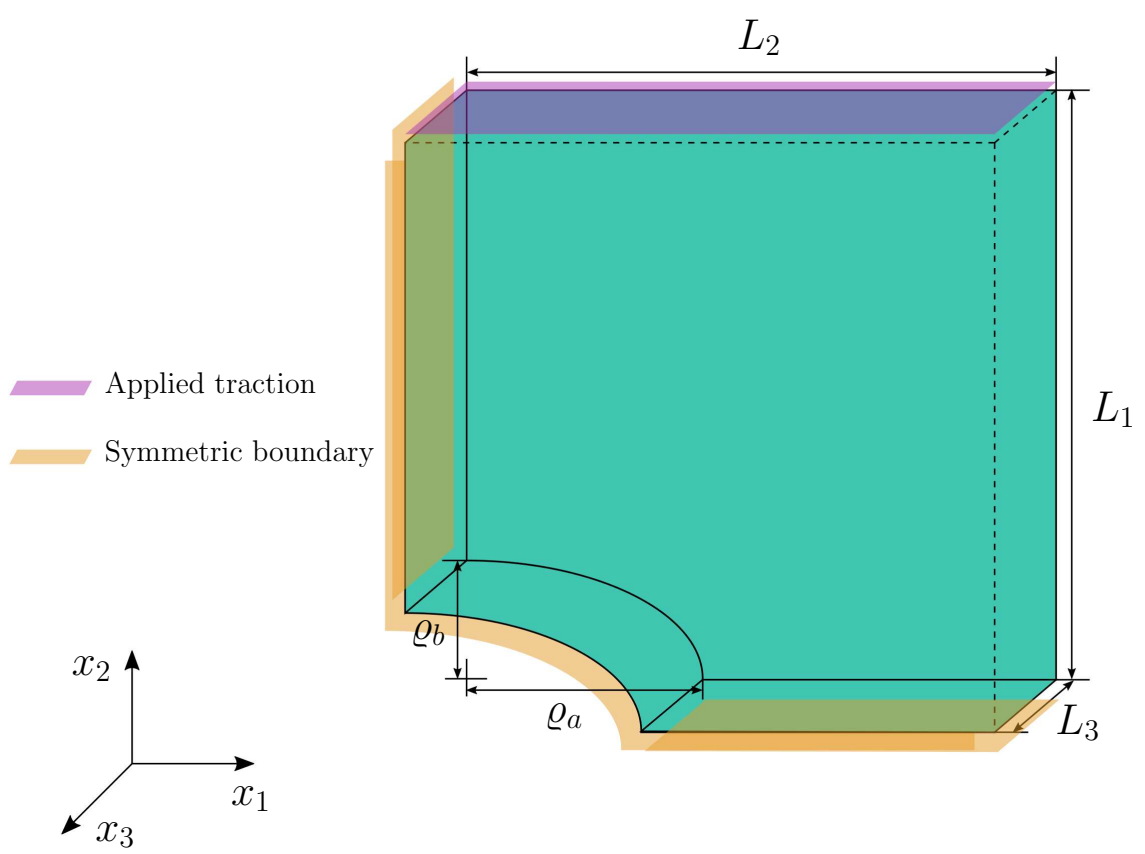

Figure 12: Block with a groove.

The structure is discretised using 120 linear 8 noded isoparametric hexahedral elements with 8 Gauss points per element. This constitutes of a total of 224 nodes and 960 Gauss points. The micro-structure is the same as used before, however now the matrix is assumed to be made of $\mathrm{Mat}_{2}$ and the fibre of Mat ${ }_{1}$.

Expected localisation of the macroscopic quantities of interest is observed as per the distribution of von Mises stress, damage and cumulative plastic strain at the end of loading, as depicted in fig. 13. More interesting is to notice their corresponding total distribution (fig. 14) at the micro-structure corresponding to the macroscopic Gauss point of maximum damage. The total von Mises stress is calculated as $\sigma_{v}=\sqrt{(3 / 2)\left(\sigma_{M}+\sigma^{*}\right)^{D}:\left(\sigma_{M}+\sigma^{*}\right)^{D}}$. It is to be mentioned that for simplicity, the macroscopic von Mises stress is calculated using $\sigma_{v M}=\sqrt{(3 / 2) \sigma_{M}^{D}: \sigma_{M}^{D}}$, and not as an average of $\sigma_{v}$. Although the maximum stress exists in the fibre, due to higher elastic limit, there is no plasticity or damage in the fibre. In the matrix region, it is observed that the distributions are not axisymmetric.

As far as the reduced basis approximation is concerned, to approximate $\varepsilon^{p *}, \alpha^{*}, \beta^{*}, R^{*}, r^{*}, D^{*}$, two basis vectors for each of them are generated. Four basis vectors each are needed to approximate $\sigma^{*}$ and $Y^{*}$, and four to approximate $\underline{u}_{1}$.

\section{Conclusion and future perspective}

This article introduces a multi-scale method based on asymptotic theory to compute elasto-viscoplasticity coupled with damage for heterogeneous structures. Verification of the method, although performed only for one-dimensional case, depicts the accuracy of the asymptotic homogenisation theory. Its feasibility is exemplified on two three-dimensional computations on structures made of a two-phase structural material. To achieve practical gains both in CPU time and storage, a reduced order modelling approach is used to cope with memory effects stored in the micro-structural internal variables, due to its highly non-linear behaviour. 

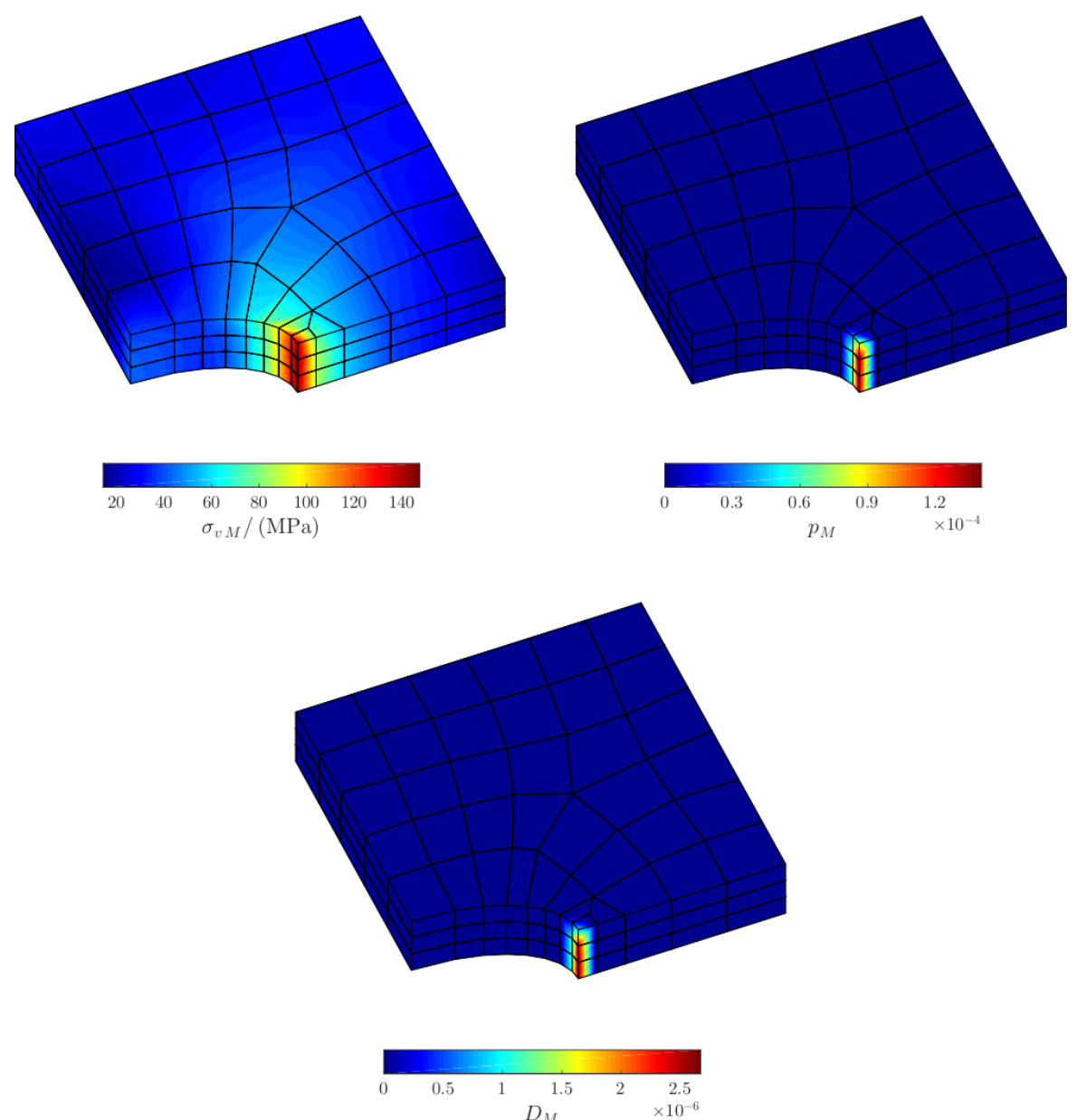

Figure 13: Distribution of macroscopic von Mises stress, damage and cumulative plastic strain at the end of loading. 

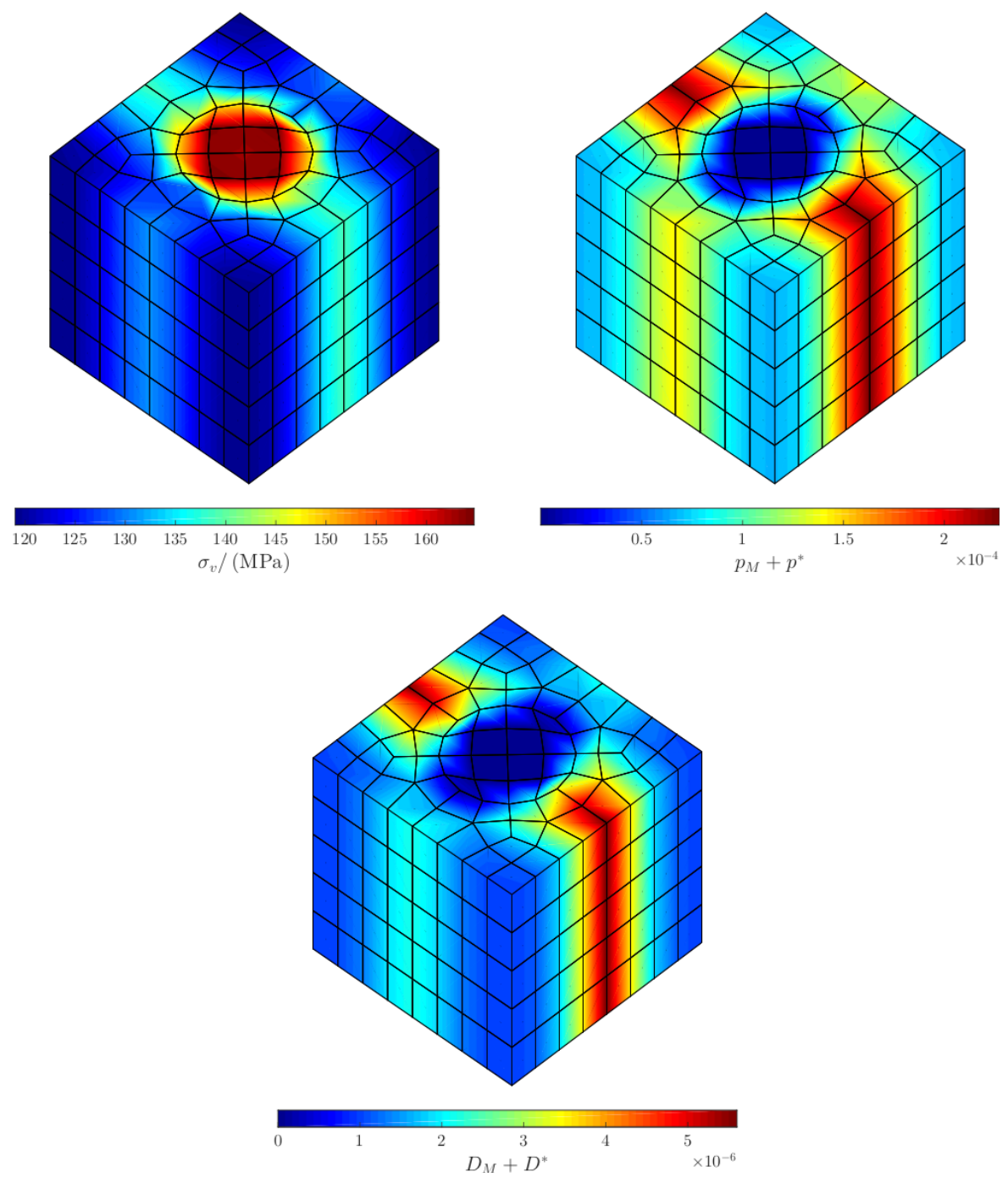

Figure 14: Distribution of total von Mises stress, damage and cumulative plastic strain at the end of loading for the weakest micro-structure. 
In the future, the scope of asymptotic homogenisation is indeed bright, as complex loads (of combined cycle fatigue type) can be simulated on a heterogeneous media using space-time homogenisation. Any edge effects, if present in the heterogeneous continua, can be adequately captured using local fine scale discretisation (local analysis or local zooming). Also in the future, the level of parallelisation can be more adequate, like nested parallelisation of the micro-structures. One important aspect that needs to be mentioned is that the numerical examples presented here are of academic nature with very coarse FE mesh. The reason behind the crude approximation is to be numerically frugal as the resolution of the non-linear constitutive behaviour can be extremely expensive. It is possible in the future to use a reduced order strategy that can avoid resolution of all the microstructures and thereby decrease numerical expense and in such cases finer level of discretisation can be used economically.

Conflict of interest. The authors declared no potential conflicts of interest with respect to the research, authorship, and/or publication of this article.

Funding. The authors disclosed receipt of the following financial support for the research, authorship, and/or publication of this article: This work was supported by the Carnot Institute Ingénierie@Lyon [project MURMUR].

\section{References}

[1] M. M. Ameen, R. H. J. Peerlings, and M. G. D. Geers. A quantitative assessment of the scale separation limits of classical and higher-order asymptotic homogenization. European Journal of Mechanics - A/Solids, 71:89-100, 2018. doi:10.1016/j.euromechsol.2018.02.011.

[2] A. Bensoussan, J.-L. Lionis, and G. Papanicolaou. Asymptotic Analysis for Periodic Structures. North Holland: Amsterdam, 1978.

[3] Mainak Bhattacharyya, Amélie Fau, Rodrigue Desmorat, Shadi Alameddin, David Néron, Pierre Ladevèze, and Udo Nackenhorst. A kinetic two-scale damage model for high-cycle fatigue simulation using multi-temporal latin framework. European Journal of Mechanics - A/Solids, 77:103808, sep 2019. doi:10.1016/j. euromechsol.2019.103808.

[4] R. Brenner and P. Suquet. Overall response of viscoelastic composites and polycrystals: exact asymptotic relations and approximate estimates. International Journal of Solids and Structures, 50(10):1824-1838, may 2013. doi:10.1016/j.ijsolstr.2013.02.011.

[5] B. Budiansky. On the elastic moduli of some heterogeneous materials. Journal of the Mechanics and Physics of Solids, 13(4):223-227, 1965. doi:10.1016/0022-5096(65)90011-6.

[6] F. Devries, H. Dumontet, G. Duvaut, and F. Lene. Homogenization and damage for composite structures. International Journal for Numerical Methods in Engineering, 27(2):285-298, 1989. doi:10.1002/nme.1620270206.

[7] David Dureisseix, Pascale Royer, and Béatrice Faverjon. Numerization of a memory effect for an homogenized composite material with a large contrast in the phase thermal conductivities. International Journal of Heat and Mass Transfer, 90:140-148, nov 2015. doi: $10.1016 / j$.ijheatmasstransfer.2015.06.048.

[8] J. D. Eshelby. The determination of the elastic field of an ellipsoidal inclusion, and related problems. Proceedings of the Royal Society of London. Series A. Mathematical and Physical Sciences, 241(1226):376-396, 1957. doi:10.1098/rspa.1957.0133.

[9] F. Feyel. Multiscale FE2 elastoviscoplastic analysis of composite structures. Computational Materials Science, 16(1):344-354, 1999. doi:10.1016/s0927-0256(99)00077-4.

[10] Frédéric Feyel and Jean-Louis Chaboche. FE2 multiscale approach for modelling the elastoviscoplastic behaviour of long fibre $\mathrm{SiC} / \mathrm{Ti}$ composite materials. Computer Methods in Applied Mechanics and Engineering, 183(3):309-330, 2000. doi:10.1016/s0045-7825(99)00224-8.

[11] Jacob Fish, Kamlun Shek, Muralidharan Pandheeradi, and Mark S. Shephard. Computational plasticity for composite structures based on mathematical homogenization: Theory and practice. Computer Methods in Applied Mechanics and Engineering, 148(1-2):53-73, 1997. doi:10.1016/ s0045-7825 (97)00030-3.

[12] Jacob Fish and Amir Wagiman. Multiscale finite element method for a locally nonperiodic heterogeneous medium. Computational Mechanics, 12(3):164-180, May 1993. doi:10.1007/ bf 00371991. 
[13] Jacob Fish, Zhiqiang Yang, and Zifeng Yuan. A second-order reduced asymptotic homogenization approach for nonlinear periodic heterogeneous materials. International Journal for Numerical Methods in Engineering, (0), 2019. doi:10.1002/nme.6058.

[14] Jacob Fish, Qing Yu, and Kamlun Shek. Computational damage mechanics for composite materials based on mathematical homogenization. International Journal for Numerical Methods in Engineering, 45(11):1657-1679, 1999. doi:10.1002/(sici) 1097-0207(19990820) 45:11<1657: : aid-nme648>3.0.co;2-h.

[15] Samuel Forest, Francis Pradel, and Karam Sab. Asymptotic analysis of heterogeneous Cosserat media. International Journal of Solids and Structures, 38(26-27):4585-4608, 2001. doi:10. 1016/s0020-7683(00)00295-x.

[16] F. Fritzen and T. Böhlke. Influence of the type of boundary conditions on the numerical properties of unit cell problems. Technische Mechanik, 30:354-363, 2010.

[17] Felix Fritzen, Samuel Forest, Thomas Böhlke, Djimedo Kondo, and Toufik Kanit. Computational homogenization of elasto-plastic porous metals. International Journal of Plasticity, 29:102-119, 2012. doi:10.1016/j.ijplas.2011.08.005.

[18] Felix Fritzen and Max Hodapp. The finite element square reduced (fe2r) method with GPU acceleration: towards three-dimensional two-scale simulations. International Journal for $\mathrm{Nu}$ merical Methods in Engineering, 107(10):853-881, 2016. doi:10.1002/nme.5188.

[19] Marc G. D. Geers, Varvara G. Kouznetsova, Karel Matous, and Julien Yvonnet. Homogenization Methods and Multiscale Modeling: Nonlinear Problems, volume Encyclopedia of Computational Mechanics Second Edition: Solids and structures, pages 1-34. John Wiley and Sons Ltd., 2017.

[20] Somnath Ghosh, Kyunghoon Lee, and Suresh Moorthy. Multiple scale analysis of heterogeneous elastic structures using homogenization theory and voronoi cell finite element method. International Journal of Solids and Structures, 32(1):27-62, 1995. doi:10.1016/0020-7683(94) 00097-g.

[21] Somnath Ghosh, Kyunghoon Lee, and Suresh Moorthy. Two scale analysis of heterogeneous elastic-plastic materials with asymptotic homogenization and Voronoi cell finite element model. Computer Methods in Applied Mechanics and Engineering, 132(1):63-116, 1996. doi:10.1016/ 0045-7825 (95) 00974-4.

[22] Huajing Guo, Bin Sun, and Zhaoxia Li. A multi-scale fatigue-creep coupled damage model for steel structures under extreme cyclic loading and temperature. International Journal of Damage Mechanics, 2019. In press. doi:10.1177/1056789519871339.

[23] J. A. Hernández, J. Oliver, A. E. Huespe, M. A. Caicedo, and J. C. Cante. High-performance model reduction techniques in computational multiscale homogenization. Computer Methods in Applied Mechanics and Engineering, 276:149-189, jul 2014. doi:10.1016/j.cma.2014.03.011.

[24] R. Hill. A self-consistent mechanics of composite materials. Journal of the Mechanics and Physics of Solids, 13(4):213-222, 1965.

[25] Tong Hui and Caglar Oskay. A high order homogenization model for transient dynamics of heterogeneous media including micro-inertia effects. Computer Methods in Applied Mechanics and Engineering, 273:181-203, 2014. doi:10.1016/j.cma.2014.01.028.

[26] V. Kouznetsova, W. A. M. Brekelmans, and F. P. T. Baaijens. An approach to micro-macro modeling of heterogeneous materials. Computational Mechanics, 27(1):37-48, Jan 2001. doi: $10.1007 / \mathrm{s} 004660000212$.

[27] V. Kouznetsova, M. G. D. Geers, and W. A. M. Brekelmans. Multi-scale constitutive modelling of heterogeneous materials with a gradient-enhanced computational homogenization scheme. International Journal for Numerical Methods in Engineering, 54(8):1235-1260, 2002. doi:10. 1002/nme. 541.

[28] E. Kröner. Zur plastischen Verformung des Vielkristalls. Acta Metall., 9:155-161, 1961.

[29] J. Lemaitre. Coupled elasto-plasticity and damage constitutive equations. Computer Methods in Applied Mechanics and Engineering, 51(1):31-49, 1985.

[30] J. Lemaitre. A course on damage mechanics. Springer, Berlin, 1996.

[31] J. Lemaitre and R. Desmorat. Engineering Damage Mechanics: Ductile, Creep, Fatigue and Brittle Failures. Springer, 2005.

[32] J. Lemaitre and I. Doghri. Damage 90: A post-processor for crack initiation. Computational Methods of Applied Mechanics Engineering, 115:197-232, 1994. doi:10.1016/0045-7825(94) 90060-4. 
[33] J. Lemaitre, J. P. Sermage, and R. Desmorat. A two scale damage concept applied to fatigue. International Journal of Fracture, 97(1-4):67-81, 1999.

[34] Matthias Leuschner and Felix Fritzen. Reduced order homogenization for viscoplastic composite materials including dissipative imperfect interfaces. Mechanics of Materials, 104:121-138, 2017. doi:10.1016/j.mechmat.2016.10.008.

[35] Xanthippi Markenscoff and Cristian Dascalu. Asymptotic homogenization analysis for damage amplification due to singular interaction of micro-cracks. Journal of the Mechanics and Physics of Solids, 60(8):1478-1485, 2012. doi:10.1016/j.jmps.2012.04.004.

[36] Kazumi Matsui, Kenjiro Terada, and Kohei Yuge. Two-scale finite element analysis of heterogeneous solids with periodic microstructures. Computers \& Structures, 82(7-8):593-606, 2004. doi:10.1016/j.compstruc.2004.01.004

[37] Christian Miehe, Jörg Schröder, and Martin Becker. Computational homogenization analysis in finite elasticity: material and structural instabilities on the micro- and macro-scales of periodic composites and their interaction. Computer Methods in Applied Mechanics and Engineering, 191(44):4971-5005, 2002. doi:10.1016/s0045-7825(02)00391-2.

[38] J. Oliver, M. Caicedo, A. E. Huespe, J. A. Hernández, and E. Roubin. Reduced order modeling strategies for computational multiscale fracture. Computer Methods in Applied Mechanics and Engineering, 313:560-595, jan 2017. doi:10.1016/j.cma.2016.09.039.

[39] Caglar Oskay and Jacob Fish. Eigendeformation-based reduced order homogenization for failure analysis of heterogeneous materials. Computer Methods in Applied Mechanics and Engineering, 196(7):1216-1243, 2007. doi:10.1016/j.cma.2006.08.015.

[40] Annika Radermacher and S. Reese. Model reduction in elastoplasticity: proper orthogonal decomposition combined with adaptive sub-structuring. Computational Mechanics, 54(3):677687, apr 2014. doi:10.1007/s00466-014-1020-6.

[41] I. B. C. M. Rocha, F. P. van der Meer, and L. J. Sluys. Efficient micromechanical analysis of fiber-reinforced composites subjected to cyclic loading through time homogenization and reduced-order modeling. Computer Methods in Applied Mechanics and Engineering, 345:644670, mar 2019. doi:10.1016/j.cma.2018.11.014.

[42] E. Sanchez-Palencia. Non-homogeneous media and vibration theory. volume 127 of Lecture Notes in Physics. Springer: Berlin, 1980.

[43] P. Suquet. Elements of homogenization for inelastic solid mechanics, volume 272, pages 193-278. Springer-Verlag, 1987.

[44] P. M. Suquet. Plasticité et homogénéisation. PhD thesis, Université Pierre et Marie Curie, Paris $6,1982$.

[45] K. Terada and N. Kikuchi. A class of general algorithms for multi-scale analyses of heterogeneous media. Computer Methods in Applied Mechanics and Engineering, 190(40-41):5427-5464, 2001. doi:10.1016/s0045-7825(01)00179-7.

[46] Y. Yang, F. Y. Ma, C. H. Lei, Y. Y. Liu, and J. Y. Li. Nonlinear asymptotic homogenization and the effective behavior of layered thermoelectric composites. Journal of the Mechanics and Physics of Solids, 61(8):1768-1783, 2013. doi:10.1016/j.jmps.2013.03.006. 